\title{
Inter-homologue repair in fertilized human eggs?
}

ARISING FROM H. Ma et al. Nature 548, 413-419 (2017); https://doi.org/10.1038/nature23305

The development and application of methods to prevent the transmission of damaging mutations through the human germ line would have considerable health benefits. In an attempt to correct a paternal pathogenic mutation using CRISPR-Cas9 technology in human embryos, Ma et al. ${ }^{1}$ assert that the maternal allele is an efficient repair template for gene correction, including when Cas 9 is applied in metaphase II (MII) oocytes. As the maternal and paternal genomes undergo distinct developmental programs and are in separate nuclei before the first mitotic division, which would seem to preclude inter-homologue interactions, we believe that it is crucial to provide a comprehensive analysis of the molecular outcomes of double-strand break (DSB) repair in human embryos. In the absence of direct molecular evidence for the inferred events, the consideration of using such methods for correction of the human germ line should proceed with extreme caution. There is a Reply to this Comment by Ma, H. et al. Nature 560, https://doi. org/10.1038/s41586-018-0381-y (2018).

Ma et al. ${ }^{1}$ use two approaches to attempt gene correction in human embryos. In one approach, which is deemed to be more promising because it is thought to give rise to non-mosaic embryos, MII oocytes were injected with donor sperm from a heterozygous mutation carrier together with Cas 9 complexes to direct the cleavage of the mutant paternal allele. About $72 \%$ of embryos arising from Cas 9 injection were thought to be wild type compared with $50 \%$ of control embryos. The authors argue that this excess of apparently wild-type embryos (22\%) arose by correction of the paternal allele, by using the maternal allele as a repair template, a process termed inter-homologue homologous recombination (abbreviated here as IH-HR).

In the other approach, Ma et al. ${ }^{1}$ again used sperm from the mutation carrier to fertilize wild-type oocytes; when the pronuclear-stage zygotes were completing S phase, they were injected with Cas9 complexes, again directed to the mutant paternal allele. In contrast to the previous approach, embryos derived from fertilization with mutant sperm could be conclusively identified because mosaic embryos were obtained. Some cells of these mosaic embryos contained a mutant paternal locus, either unmodified or with small indels, together with the wild-type maternal allele. Other cells in these mosaic embryos contained only a detectable wild-type allele. The authors inferred that these cells arose by IH-HR of the mutant paternal allele using the wild-type maternal allele as a template, leading to gene correction.

Considering the data presented in Ma et al. ${ }^{1}$, alternatives to IH-HR are possible. Genotyping involved the amplification of an approximately 534-base-pair (bp) fragment in which the MYBPC $3^{\triangle G A G T}$ mutation is approximately $200 \mathrm{bp}$ from one of the primer-binding sites. Deletions larger than $200 \mathrm{bp}$ would be sufficient to remove this primer-binding site and lead to amplification of only the maternal allele (Fig. 1a, b), giving the misleading appearance of gene correction of the paternal allele. Although typically not as common as small indels, long deletions and other events have been detected in cultured cells and in both mouse and pig zygotes ${ }^{2-4}$. To detect longer deletions, a matrix of primer pairs needs to be tiled at increasing distances from both sides of the mutation; linkage analysis performed on the long-range PCR products would confirm whether amplification is from both the maternal and the paternal chromosomes. In a study designed to score these events systematically, Cas9-induced double-strand breaks in mouse embryonic stem cells were found to resolve into large deletions (250-9,500 bp) in approximately $20 \%$ of edited cells ${ }^{5}$. This approach remains imperfect to detect all events, however, because very large deletions or other events such as inversions, translocations, chromosome loss and large insertions prevent amplification and thus will escape characterization. Indeed, in 19\% of cells edited at an autosomal locus, only one of two alleles could be recovered ${ }^{5}$. These various outcomes of repair of a DSB could result in genotypes incompatible with normal development, and therefore need to be reliably identified to exclude affected embryos.

Wild-type genotypes in a PCR assay can also arise by the activation of the egg during Cas9 injection, but without successful integration of a sperm genome, resulting in haploid or diploid parthenogenetic cells containing only the maternal genome ${ }^{6}$ (Fig. 1c). A paternal contribution was verified by cytogenetic analysis in some of the stem-cell lines generated from embryos by Ma et al. ${ }^{1}$, but the authors did not determine whether wild-type stem-cell lines were from wild-type sperm, or arose by gene correction.

To directly demonstrate gene correction by IH-HR, evidence for a new linkage of maternal and paternal alleles-that is, through the incorporation of the wild-type sequence from one of the maternal homologues into the mutant paternal chromosome at the site of the DSB - is required (Fig. 1a). New DNA linkages can be determined by phased DNA sequencing, or by long-range PCR using allele-specific primers $^{7,8}$. Such haplotype analysis is particularly crucial in the case of the embryos derived from MII-phase oocyte injections, because which embryos were derived from sperm carrying the mutant allele was not determined.

Although IH-HR in fertilized oocytes and zygotes cannot be excluded, there are several obstacles to this mechanism. IH-HR after the induction of a DSB in mitotic mammalian cells has been described ${ }^{11}$, and was also seen in a recent study using CRISPR-Cas9 in embryonic stem cells ${ }^{5}$, though it was less frequent than inter-sister HR or NHEJ. In mammals, IH-HR is essential for the reductional division to form gametes, and is promoted by the large number of DSBs that are programmed to form on each chromosome ${ }^{9}$. It is important to note, however, that meiotic IH-HR occurs during fetal development in females ${ }^{10}$ and so it is temporally removed from the events described in Ma et al ${ }^{1}$.

The physical separation of maternal and paternal genomes in fertilized eggs would be expected to be a substantial impediment to IH-HR during the first cell cycle. After fertilization, distinct maternal and paternal nuclei form (pronuclei), such that the two genomes are separate in a cell that is more than $100 \mu \mathrm{m}$ in diameter (Fig. 1d-g). This separation may prevent the incorporation of paternal chromosomes into the oocyte MII spindle (Fig. 1e). During the first interphase, maternal and paternal pronuclei migrate from the site of their formation towards the centre of the zygote, but their integrity persists throughout interphase (Fig. 1f, g), during which individual nuclei can be manipulated ${ }^{12}$. In both human and mouse zygotes, maternal and paternal genomes undergo DNA replication in separate nuclei, and enter the first mitosis as separate entities, at which time they can still be individually manipulated (Fig. 1f, g). Microtubule action assembles maternal and paternal genomes on a common metaphase plate at the 
a
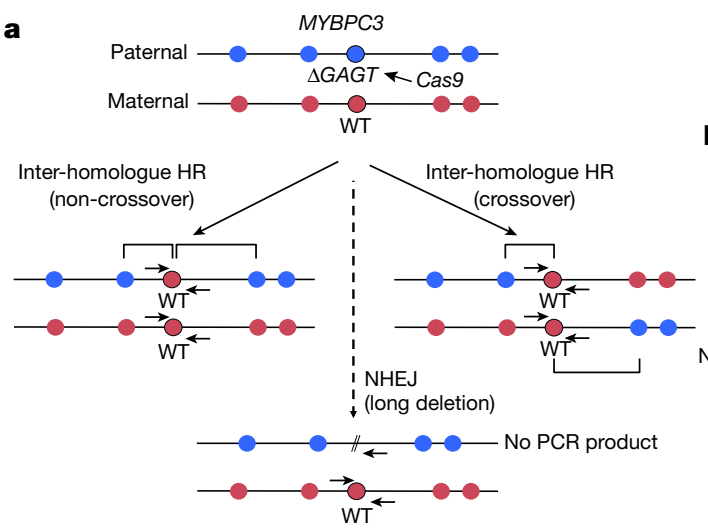

b

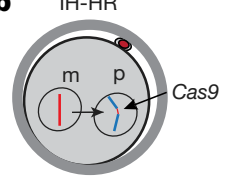

NHEJ (long deletion)

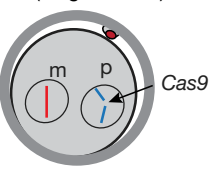

d

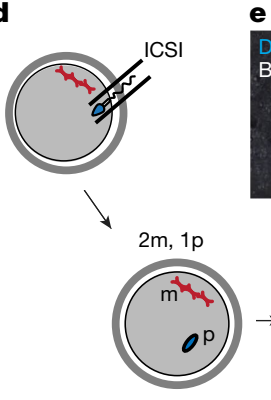

g
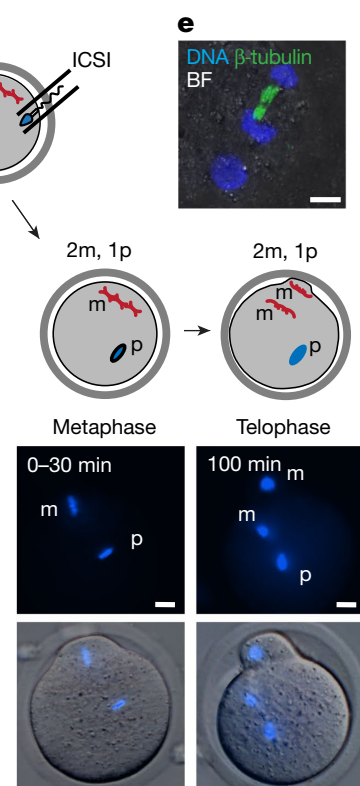

f Interphase

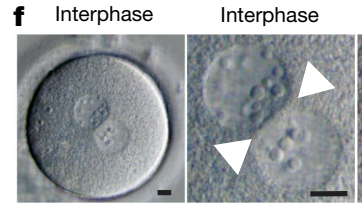

$2 \mathrm{~m}, 1 \mathrm{p}$

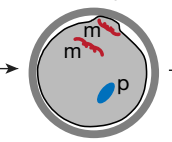

Telophase
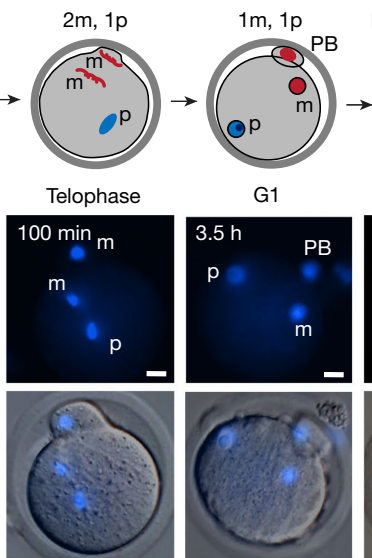

G1
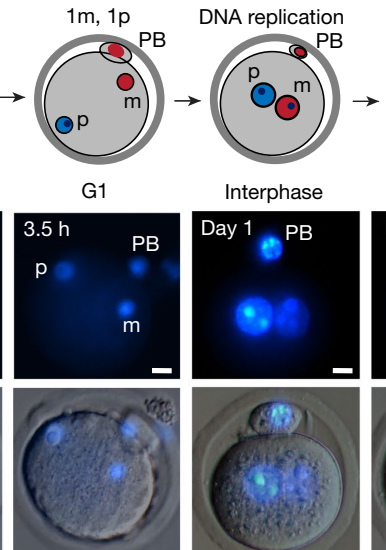

Interphase
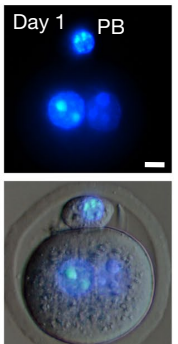

C

Maternal only
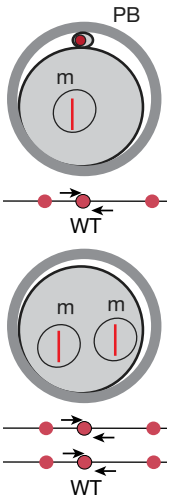

Prometaphase
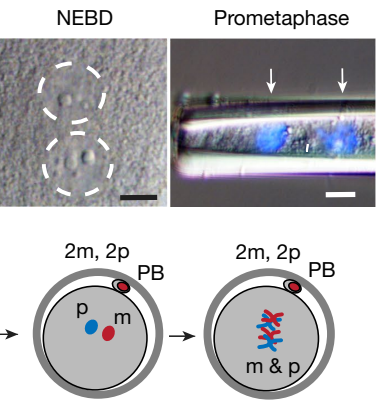

Prometaphase

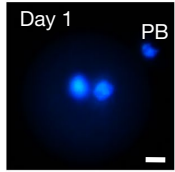

Metaphase

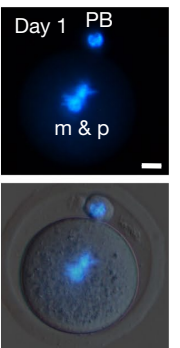

Fig. 1 Constraints on gene editing by inter-homologue homologous recombination (IH-HR) in the early human embryo. a, Possible repair outcomes after a Cas9-induced DSB at the paternal $M Y B P C 3^{\triangle G A G T}$ locus. Red and blue circles indicate unique maternal and paternal genetic variants, respectively. IH-HR results in gene conversion of the paternal allele by the wild-type (WT) maternal allele. The repair outcome can be a non-crossover or a crossover. Only one outcome of crossing-over is shown, in which the recombined chromosomes segregate to the same nucleus. The alternative is that the recombined chromosomes segregate to different daughter cells, such that loss of heterozygosity would occur on the chromosome from the point of the IH-HR event to the telomere in both daughter cells, one with homozygosity for the maternal chromosome and the other for the paternal chromosome. This outcome would be expected in half of the crossing-over events that underwent IH-HR in the G2 phase of the cell cycle. NHEJ can lead to small indels (not shown), but events are also possible that result in the deletion of a primer-binding site used for genotyping (as shown). b, Schematic of possible repair outcomes after Cas9 cleavage in the human zygote from panel a. m, maternal chromosome; p, paternal chromosome. c,

first mitosis ${ }^{13,14}$, although they remain in distinct groups ${ }^{15}$. Therefore, direct contact between maternal and paternal genomes required for inter-homologue repair does not seemingly occur until the first mitosis or later when embryos enter the two-cell stage and the two genomes are packaged within the same nucleus. Relative to the application of Cas9, this is $24-30 \mathrm{~h}$ after the MII injections, and 6-12 h after the zygotic injections.

It is important to note the different outcomes Ma et al. ${ }^{1}$ obtained with regards to mosaicism depending on whether CRISPR-Cas9 was
Parthenogenesis after fertilization failure with (top) and without (bottom) second polar body (PB) extrusion. Outcomes of $\mathbf{a}-\mathbf{c}$ are indistinguishable in genotyping assays using flanking PCR primers alone. d, Schematic of intracytoplasmic sperm injection (ICSI) followed by progression through the first cell cycle during day 1 of development. The number of maternal and paternal genomes is indicated at each phase of the cell cycle. e, Immunofluorescence of a mouse zygote at telophase of the second maternal meiotic division. Note that only the maternal genomes are attached to microtubules, while the paternal genome begins to form an interphase nuclear membrane to replace the sperm membrane. BF, bright field. f, Progression of human zygotes through the first cell cycle from the two-pronuclear stage to prometaphase, when the two genomes can be removed from the egg by a needle. Note the separation of the two genomes (arrows and dashed circles). NEBD, pronuclear envelope breakdown. g, Cell cycle progression during day 1 in fertilized mouse zygotes. Of 23 mouse eggs, none showed direct contact between the maternal and paternal genomes. Scale bars, $10 \mu \mathrm{m}$. Images one and four (from the left) in $\mathbf{f}$ are from Egli et al. ${ }^{14}$; images four to six (from the left, top) in $\mathbf{g}$ are from Egli et al. ${ }^{13}$.

injected into zygotes (frequent mosaicism) or together with sperm into MII-phase eggs (lack of apparent mosaicism). Mosaicism from zygotic injection is consistent with DSB repair occurring after DNA replication, whereas the lack of mosaicism after MII-phase injection indicates that repair occurs before DNA replication and thus before the first mitosis. Consistent with this, the injection of CRISPR-Cas9 together with sperm in mouse MII oocytes results in non-mosaic modification of the paternal genome within only $3 \mathrm{~h}$ owing to NHEJ during sperm 
chromatin decondensation ${ }^{16}$. Notably, the maternal genome seems to be refractory to editing during this time of meiotic exit. Different outcomes depending on the time of CRISPR-Cas9 injection indicate that gene editing in the paternal genome during decondensation or after the formation of a nucleus may involve different repair mechanisms.

In summary, the direct verification of gene correction and exclusion of other possible outcomes is an imperative for any embryo that would be considered for future implantation. Gene editing has the potential to reduce disease-causing alleles, but inadvertent changes to the human germ line, including rearrangements, long deletions, and loss of heterozygosity, for example, from IH-HR, could have serious consequences that affect development, predisposition to cancer and fertility. Our discussion of Ma et al. ${ }^{1}$ demonstrates the need for a more comprehensive characterization of the repair mechanisms in the early embryo, and identifies a key challenge for the therapeutic use of gene editing in the human germ line: the development of reliable assays to distinguish between different repair outcomes when DNA is limiting.

\section{Methods}

Mouse oocytes were obtained from 5-7-week-old B6D2F1/J females (Jackson Laboratories stock 10006) by superovulation. Oocytes were removed from oviducts $14 \mathrm{~h}$ after injection of human chorionic gonadotropin, and fertilized with mouse sperm injection by ICSI on an inverted Olympus IX73 equipped with a Narishige micromanipulator. Images were taken using an Olympus IX73 equipped with an Olympus DP80 camera (Fig. 1f) or a Zeiss 710 confocal microscope at indicated time points (Fig. 1e). Immunostaining was performed using a monoclononal beta tubulin antibody (clone AA2, Millipore 05-661, dilution 1:1,000, lot 2370698 ) in PBS with $10 \% \mathrm{FBS}$ for $3 \mathrm{~h}$ at room temperature. Secondary antibody Invitrogen 488 donkey anti mouse (A21202) at 1:500 dilution in PBS for 45 min at room temperature. Hoechst 33342 was used for DNA staining at $5 \mu \mathrm{g} \mathrm{ml}^{-1}$ (Life Technologies H3570). All animal research was reviewed and approved by the Columbia University IACUC, and performed in accordance with animal use guidelines and applicable ethical regulations.

Data availability. All available data are included in this manuscript and available from the corresponding authors upon reasonable request.

\section{Dieter Egli ${ }^{1}$, Michael V. Zuccaro ${ }^{2}$, Michael Kosicki ${ }^{3}$, \\ George M. Church ${ }^{4}$, Allan Bradley ${ }^{3}$ \& Maria Jasin ${ }^{5 *}$}

${ }^{1}$ Department of Obstetrics and Gynecology and Department of Pediatrics, Columbia University, New York, NY, USA. ${ }^{2}$ Graduate Program, Department of Physiology and Cellular Biophysics, Columbia University, New York, NY, USA. ${ }^{3}$ Wellcome Sanger Institute, Hinxton, Cambridge, UK. ${ }^{4}$ Department of Genetics, Harvard Medical School, Boston, MA, USA. ${ }^{5}$ Developmental Biology Program, Memorial Sloan Kettering Cancer Center, New York, NY, USA.*e-mail: de2220@cumc.columbia.edu; m-jasin@ski.mskcc.org

Received: 26 August 2017; Accepted: 5 April 2018.
1. Ma, H. et al. Correction of a pathogenic gene mutation in human embryos. Nature 548, 413-419 (2017).

2. Shin, H. Y. et al. CRISPR/Cas9 targeting events cause complex deletions and insertions at 17 sites in the mouse genome. Nat. Commun. 8, 15464 (2017).

3. Whitworth, K. M. et al. Use of the CRISPR/Cas9 system to produce genetically engineered pigs from in vitro-derived oocytes and embryos. Biol. Reprod. 91, 78 (2014).

4. Parikh, B. A., Beckman, D. L., Patel, S. J., White, J. M. \& Yokoyama, W. M. Detailed phenotypic and molecular analyses of genetically modified mice generated by CRISPR-Cas9-mediated editing. PLoS One 10, e0116484 (2015).

5. Kosicki, M., Tomberg, K. \& Bradley, A. Repair of double-strand breaks induced by CRISPR-Cas9 leads to large deletions and complex rearrangements. Nat. Biotechnol. https://doi.org/10.1038/nbt.4192 (2018).

6. Sultan, K. M., Munné, S., Palermo, G. D., Alikani, M. \& Cohen, J. Chromosomal status of uni-pronuclear human zygotes following in-vitro fertilization and intracytoplasmic sperm injection. Hum. Reprod. 10, 132-136 (1995).

7. Cole, F. et al. Mouse tetrad analysis provides insights into recombination mechanisms and hotspot evolutionary dynamics. Nat. Genet. 46, 1072-1080 (2014).

8. Jeffreys, A. J. \& May, C. A. Intense and highly localized gene conversion activity in human meiotic crossover hot spots. Nat. Genet. 36, 151-156 (2004).

9. Kauppi, L. et al. Numerical constraints and feedback control of double-strand breaks in mouse meiosis. Genes Dev. 27, 873-886 (2013).

10. Baker, T. G. A quantitative and cytological study of germ cells in human ovaries. Proc. R. Soc. Lond. B 158, 417-433 (1963)

11. Stark, J. M. \& Jasin, M. Extensive loss of heterozygosity is suppressed during homologous repair of chromosomal breaks. Mol. Cell. Biol. 23, 733-743 (2003).

12. Kattera, S. \& Chen, C. Normal birth after microsurgical enucleation of tripronuclear human zygotes: case report. Hum. Reprod. 18, 1319-1322 (2003).

13. Egli, D., Rosains, J., Birkhoff, G. \& Eggan, K. Developmental reprogramming after chromosome transfer into mitotic mouse zygotes. Nature 447, 679-685 (2007).

14. Egli, D. et al. Reprogramming within hours following nuclear transfer into mouse but not human zygotes. Nature Commun. 2, 488 (2011).

15. Reichmann, J. et al. Dual-spindle formation in zygotes keeps parental genomes apart in early mammalian embryos. Science $\mathbf{3 6 1}, 189-193$ (2018).

16. Suzuki, T., Asami, M. \& Perry, A. C. Asymmetric parental genome engineering by Cas9 during mouse meiotic exit. Sci. Rep. 4, 7621 (2014).

Author contributions D.E. and M.J. wrote the paper with contributions from M.K., A.B., M.Z. and G.M.C. M.Z. and D.E. performed ICSI and imaging of eggs. D.E. is a NYSCF-Robertson investigator.

Competing interests G.M.C. is an advisor at Veritas Genetics and Editas Medicine. A comprehensive list of G.M.C.'s technology transfer and advisory roles is provided here http://arep.med.harvard.edu/gmc/tech.html. Other authors declare no competing interests.

\section{Additional information}

Supplementary information accompanies this Comment. Reprints and permissions information is available at http://www.nature.com/ reprints.

Correspondence and requests for materials should be addressed to D.E. or M.J.

https://doi.org/10.1038/s41586-018-0379-5 


\section{Reporting Summary}

Nature Research wishes to improve the reproducibility of the work that we publish. This form provides structure for consistency and transparency in reporting. For further information on Nature Research policies, see Authors \& Referees and the Editorial Policy Checklist.

\section{Statistical parameters}

When statistical analyses are reported, confirm that the following items are present in the relevant location (e.g. figure legend, table legend, main text, or Methods section).

n/a Confirmed

$\square \searrow$ The exact sample size $(n)$ for each experimental group/condition, given as a discrete number and unit of measurement

$\bigotimes \square$ An indication of whether measurements were taken from distinct samples or whether the same sample was measured repeatedly

\ $\square$ The statistical test(s) used AND whether they are one- or two-sided

Only common tests should be described solely by name; describe more complex techniques in the Methods section.

Х $\square$ A description of all covariates tested

$\bigotimes \square$ A description of any assumptions or corrections, such as tests of normality and adjustment for multiple comparisons

Х $\square$ A full description of the statistics including central tendency (e.g. means) or other basic estimates (e.g. regression coefficient) AND

$\bigotimes \square$ variation (e.g. standard deviation) or associated estimates of uncertainty (e.g. confidence intervals)

$\bigotimes \square$ For null hypothesis testing, the test statistic (e.g. $F, t, r$ ) with confidence intervals, effect sizes, degrees of freedom and $P$ value noted

$\triangle$ Give $P$ values as exact values whenever suitable.

$\bigotimes \square$ For Bayesian analysis, information on the choice of priors and Markov chain Monte Carlo settings

\ $\square$ For hierarchical and complex designs, identification of the appropriate level for tests and full reporting of outcomes

$\bigotimes \square$ Estimates of effect sizes (e.g. Cohen's $d$, Pearson's $r$ ), indicating how they were calculated

$\bigotimes \square$ Clearly defined error bars

$\square$ State explicitly what error bars represent (e.g. SD, SE, CI)

Our web collection on statistics for biologists may be useful.

\section{Software and code}

Policy information about availability of computer code
Data collection
no software was used for data collecdtion
Data analysis
no software was used for data analysis

For manuscripts utilizing custom algorithms or software that are central to the research but not yet described in published literature, software must be made available to editors/reviewers upon request. We strongly encourage code deposition in a community repository (e.g. GitHub). See the Nature Research guidelines for submitting code \& software for further information.

\section{Data}

Policy information about availability of data

All manuscripts must include a data availability statement. This statement should provide the following information, where applicable:

- Accession codes, unique identifiers, or web links for publicly available datasets

- A list of figures that have associated raw data

- A description of any restrictions on data availability 


\section{Field-specific reporting}

Please select the best fit for your research. If you are not sure, read the appropriate sections before making your selection.

$\bigotimes$ Life sciences $\quad \square$ Behavioural \& social sciences

For a reference copy of the document with all sections, see nature.com/authors/policies/ReportingSummary-flat.pdf

\section{Life sciences}

\section{Study design}

All studies must disclose on these points even when the disclosure is negative.

Sample size the sample size was chosen based on the interpretation in the Ma et al. paper that about half (50\%) of the eggs undergo interhomolog repair after a double strand break, requiring interaction between maternal and paternal nuclei. We found none interacted of 23 . We calculated that with just five samples, we would have reached statistical significance that our observations were different than Ma et al. Our sample size meets and exceeds statistical requirements.

Data exclusions all data presented is available in the manuscript. No exclusion.

Replication all attempts 23 of 23 yielded the same result

Randomization randomization cannot be applied here as a single condition is evaluated

Blinding blinding can not be done with a single condition

\section{Materials \& experimental systems}

Policy information about availability of materials
$\mathrm{n} / \mathrm{a}$ Involved in the study
\ $\square$ Unique materials
$\square$ Antibodies
Х $\square$ Eukaryotic cell lines
$\square$ Research animals
Х $\square$ Human research participants

Antibodies

Antibodies used

beta tubulin Upstate/Millipore, 05-661, dilution 1:1000, lot \#2370698

Validation

Validation statement on company web site: "This antibody recognizes $\beta$-Tubulin isotypes I, II, III, IVa, and IVb of $50 \mathrm{kDa}$. No cross-reactivity with alpha or gamma tubulin." Our own validation is that the antibody stains the structure with the expected morphology of the microtubule spindle. No such staining is found in cells at interphase of the cell cycle. This same antibody has been used in previous publications. Yamada, M. and Egli, D. (2017). Genome Transfer Prevents Fragmentation and Restores Developmental Potential of Developmentally Compromised Postovulatory Aged Mouse Oocytes. Stem Cell Reports 8, 576-588.

Research animals

Policy information about studies involving animals; ARRIVE guidelines recommended for reporting animal research

Animals/animal-derived materials 5-7 week old B6D2F1/J female and male mice (Jackson Laboratories stock \# 10006)

\section{Method-specific reporting}

n/a $\mid$ Involved in the study

X $\square$ ChIP-seq

Х $\square$ Flow cytometry

$\backslash \square$ Magnetic resonance imaging 


\section{BRIEF COMMUNICATIONS ARISING}

\section{Large deletions induced by Cas 9 cleavage}

ARISING FROM H. Ma et al. Nature 548, 413-419 (2017); https://doi.org/10.1038/nature23305

In a recent publication, $\mathrm{Ma}$ et al. ${ }^{1}$ reported the correction of a heterozygous paternally inherited MYBPC3 mutation in human zygotes using CRISPR-Cas9 genome editing. We read their work with interest, especially the interpretation that the wild-type maternal allele was used as template to repair the DNA break at the mutation site. We think there are other explanations, specifically that repair of CRISPRCas9 single cleavage at the mutation site generates large deletions that prevent PCR amplification of the paternal chromosome, thereby giving the appearance of inter-homologous repair. Ma et al. ${ }^{1}$ did not perform experiments to exclude this possibility, and we therefore sought to test this idea in mouse embryos, which closely model the development of human zygotes. There is a Reply to this Comment by $\mathrm{Ma}, \mathrm{H}$. et al. Nature 560, https://doi.org/10.1038/s41586-018-0381-y (2018).

Similar to Ma et al. ${ }^{1}$, we delivered the CRISPR reagents into zygotes using microinjection. We used Cas 9 mRNA as opposed to CAS9 protein, as this approach has been shown to generate on-target mutations with high efficiency ${ }^{2}$. Guide RNAs (gRNAs) were identified for six autosomal loci. Three of the gRNAs targeted coding regions of genes that do not cause nullizygous lethality (Rsad2 (also known as viperin), Pik3r6 and Hmgcs2) and three targeted intronic or flanking regions (Neurog3 (also known as Ngn3), Foxp4 and Fzd3). Altogether, 127 founder embryos/mice were generated from zygotes microinjected with each gRNA. To assess the mutation efficiency at each locus, we used the polyacrylamide gel heteroduplex mobility assay (HMA) ${ }^{3}$ to screen 300-600 base-pair (bp) PCR products that span the gRNA target sites. The HMA detected mutations in 76 out of $127(60 \%)$ of samples (Supplementary Information tab 1). These included 13 samples that generated considerably smaller PCR products than expected, indicating that they contain large deletions in the order of 100-300 bp (Supplementary Information tab 1). Notably, four samples failed to amplify, suggesting bialleic deletion of PCR primer-binding sites (Supplementary Information tab 1). To confirm the results of the HMA, we performed Sanger sequencing of the PCR products. As expected, mutations were detected in all HMA-positive samples (Supplementary Information tab 2). Notably, 93\% of the HMA-negative samples that we sequenced contained a small indel compared with the wild-type sequence (Supplementary Information tab 3). Only one type of mutation was observed in most of these samples, which explained why they were not detected by HMA (false negatives). Taken together, these data show that mutations were generated in $98 \%$ of CRISPR-Cas9microinjected zygotes.

Next, we screened specifically for large deletions using an approximately 1.6-kb PCR with primers that were equidistant from gRNA PAM sequences. Notably, we generated amplicons that were considerably less than $1.6 \mathrm{~kb}$ (indicating large deletions) in 35 samples. These low molecular mass products were not generated in the initial 300-600-bp PCR, indicating that they corresponded to deletions that encompass at least one of the (initial) PCR primer-binding sequences. Large deletion products were also identified in 9 additional samples when we performed an approximately 3.2-kb PCR on the Neurog3 and Foxp4 founders (Supplementary Information tab 4). These larger PCRs also generated amplicons from samples that failed to amplify in the initial 300-600-bp PCR, confirming that these contained large deletion alleles. Altogether, the number of samples containing detectable large deletions (more than $100 \mathrm{bp)}$ ) was 57 out of 127 (45\%; Fig. 1a, Supplementary Information tab 4), noting that some founders were mosaic and some contained more than one large deletion event. Large deletions were detected in $57 \%$ of the HMA-negative samples, indicating that these large deletions contributed to the amplification failure in the HMA false-negative samples. The remaining mutant HMA-negative samples may contain larger deletions that require further separation of PCR primers for detection, or may be homozygous for the indel mutation. Deleted sequences were confirmed by direct sequencing of gelpurified PCR products (Supplementary Information tab 5). Each gRNA generated a range of unique deletions, indicating that the process that underpins the generation of large deletions is stochastic (Fig. 1b, Supplementary Information tab 5). Notably, we observed that the orientation of the large deletions was asymmetric or unidirectional with respect to the cutting site (Fig. 1b, Supplementary Information tab 5). The size of the large deletions (after 1.6-kb PCR) ranged from 100 to $800 \mathrm{bp}$ (Fig. 1b), with the largest deletion of $2.3 \mathrm{~kb}$ detected in a Foxp 4 sample after 3.2-kb PCR (Supplementary Information tabs 4 and 5, sample Foxp4 \#19).

Because the detection of large deletions using PCR is prone to amplification bias and is confounded by the deletion of primer sequences, we next sought to use an unbiased approach using PCR-free pairedend whole-genome sequencing (WGS) to determine the frequency and extent of large deletions in CRISPR-Cas9-treated mouse embryonic stem (ES) cells. We transfected ES cells with plasmid PX459.V2.0 ${ }^{4}$ that expresses Cas 9 and Rsad2 gRNA, and puromycin-resistant transfectants were obtained for genomic DNA (gDNA) extraction and WGS analysis. Of 88 sequence reads that span the Rsad 2 single gRNA cleavage site, only two $(2.3 \%)$ corresponded to wild-type alleles. Small indels and substitutions were found in 33 reads (37.5\%), and large deletions inferred from discordant mapping of paired-end reads and split read mapping over the breakpoint were detected in 33 reads $(37.5 \%)$ (Fig. 1c). The remaining 20 reads (23\%) unexpectedly contained insertions of the PX459.V2.0 expression plasmid. Together, these data confirm that large deletions are frequently generated after CRISPRCas9-mediated DNA cleavage in mouse ES cells and zygotes.

In summary, our data demonstrate that large deletions are frequently generated in mouse zygotes after CRISPR-Cas9 single cleavage, as has recently been noted by others ${ }^{5-7}$. Although species differences may affect DNA repair products, Ma et al. ${ }^{1}$ cannot conclude with certainty that the purported homology-directed repair gene correction event has generated homozygous wild-type embryos until the existence of large deletions is excluded. This could be investigated by generating larger PCR products as described above. Quantitative PCR analysis to confirm the presence of both wild-type alleles would provide definitive evidence. The importance of accurate genotyping in the context of human germ-line modification cannot be overstated. Failure to detect large deletions could lead to disastrous outcomes in potential clinical applications.

\section{Methods}

gRNAs were designed using the online CRISPR tool developed by the Zhang laboratory at MIT (http://crispr.mit.edu) ${ }^{8}$. Cas 9 mRNA $\left(100 \mathrm{ng} \mu \mathrm{l}^{-1}\right)$ and gRNAs (50 ng $\mathrm{Il}^{-1}$ each) were injected into the cytoplasm of C57BL/6N zygotes using a FemtoJet microinjector, transferred to pseudo-pregnant recipients, and allowed to develop to term or collected as embryos. gRNA sequences were as follows: Neurog $35^{\prime}$-GCACAGCTGGATTCCGGACAAA- $3^{\prime}$; Foxp 4 5'-CCAGCGTTCCCATTGTCCTT-3'; Fzd3 5'-CTTAGCAAGG 
Large deletions detected $\square$ Large deletions undetected

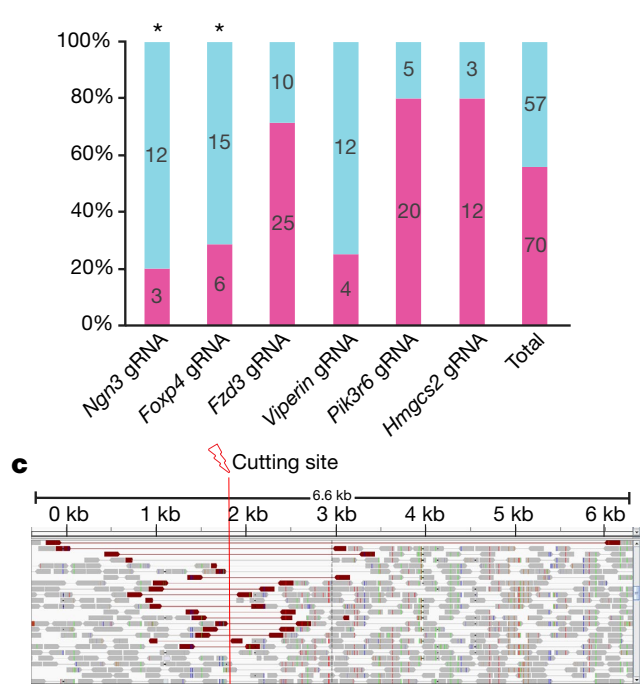

Fig. 1 | Frequent large deletions detected in mouse zygote injections. a, The number of founders that contain large deletions detected by large PCR $(\sim 1.6 \mathrm{~kb})$. Asterisk symbol indicates $\sim 3.2-\mathrm{kb}$ PCR was also performed. $\mathbf{b}$, Sequencing of large deletion bands after $\sim 1.6-\mathrm{kb}$ PCR from mouse zygote injection samples. '0' represents the cutting site of Cas9. b

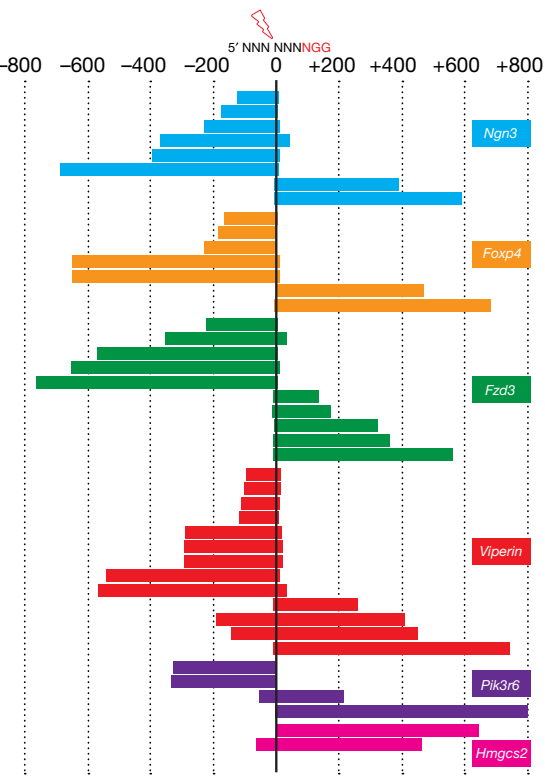

Each bar represents the deletion position relative to the corresponding NGG PAM sequence. c, Whole-genome sequencing analysis of Rsad2gRNA-treated mouse ES cell pools. Integrative genome viewer snapshot shows reads paired in sequencing and sorted by insert size. Read pairs in red indicate discordantly mapping pairs indicative of large deletions.
GTGTGAAAAG- $3^{\prime} ;$ Rs $a d 2$ 5'-GGGTGGCTAGATCCCGGGA-3'; Pik3r6 $5^{\prime}$-CTTACCCTGATTGCTCTGGA-3'; and Hmgcs $25^{\prime}$-TACAATCCCTCCTG CTCCCC- ${ }^{\prime}$. All animal work was conducted following approval by The University of Adelaide Animal Ethics Committee in accordance with the Australian code for the care and use of animals for scientific purposes.

Data availability. All data and reagents are available from the corresponding author upon request.

\section{Fatwa Adikusuma ${ }^{1,2,3,4}$, Sandra Piltz ${ }^{1,2,4}$, Mark A. Corbett ${ }^{2}$, Michelle Turvey ${ }^{5}$, Shaun R. McColl ${ }^{1}$, Karla J. Helbig ${ }^{6}$, Michael R. Beard ${ }^{1}$, James Hughes ${ }^{1}$, Richard T. Pomerantz ${ }^{7}$ \& Paul Q. Thomas ${ }^{1,2,4 *}$}

${ }^{1}$ School of Biological Sciences, University of Adelaide, Adelaide, South Australia, Australia. ${ }^{2}$ Robinson Research Institute, The University of Adelaide, Adelaide, South Australia, Australia. ${ }^{3}$ Centre for Biomedical Research (CEBIOR), Faculty of Medicine, Diponegoro University, Semarang, Indonesia. ${ }^{4}$ South Australian Health and Medical Research Institute, Adelaide, South Australia, Australia. ${ }^{5}$ Singapore-MIT Alliance for Research and Technology, Singapore, Singapore. ${ }^{6}$ School of Life Sciences, La Trobe University, Melbourne, Victoria, Australia. ${ }^{7} \mathrm{Fels}$ Institute for Cancer Research, Department of Medical Genetics and Molecular Biochemistry, Temple University Lewis Katz School of Medicine, Philadelphia, PA, USA.*e-mail: paul.thomas@adelaide.edu.au

Received: 29 August 2017; Accepted: 5 April 2018.

1. Ma, $\mathrm{H}$. et al. Correction of a pathogenic gene mutation in human embryos. Nature 548, 413-419 (2017).
2. Wang, $\mathrm{H}$. et al. One-step generation of mice carrying mutations in multiple genes by CRISPR/Cas-mediated genome engineering. Cell 153, 910-918 (2013).

3. Chen, J. et al. Efficient detection, quantification and enrichment of subtle allelic alterations. DNA Res. 19, 423-433 (2012).

4. Ran, F. A. et al. Genome engineering using the CRISPR-Cas9 system. Nat. Protocols 8, 2281-2308 (2013).

5. Shin, H. Y. et al. CRISPR/Cas9 targeting events cause complex deletions and insertions at 17 sites in the mouse genome. Nat. Commun. 8, 15464 (2017).

6. Parikh, B. A., Beckman, D. L., Patel, S. J., White, J. M. \& Yokoyama, W. M. Detailed phenotypic and molecular analyses of genetically modified mice generated by CRISPR-Cas9-mediated editing. PLoS One 10, e0116484 (2015)

7. Zuckermann, M. et al. Somatic CRISPR/Cas9-mediated tumour suppressor disruption enables versatile brain tumour modelling. Nat. Commun. 6, 7391 (2015).

8. Cong, L. et al. Multiplex genome engineering using CRISPR/Cas systems Science 339, 819-823 (2013).

Author contributions F.A. and P.Q.T. conceived and designed the study. F.A. performed all experiments apart from zygote microinjections (performed by S.P.), the heteroduplex assay and sequencing of Rsad2, Pik3r6 and Hmgcs2 mice (performed by K.J.H., M.R.B., M.T., S.R.M. and J.H.) and WGS data analysis (performed by M.C.). F.A., R.T.P. and P.Q.T. wrote the manuscript with the input from all authors.

Competing interests Declared none.

Additional information

Supplementary information accompanies this Comment.

Reprints and permissions information is available at http://www.nature.com/ reprints.

Correspondence and requests for materials should be addressed to P.Q.T.

https://doi.org/10.1038/s41586-018-0380-z 


\section{Reporting Summary}

Nature Research wishes to improve the reproducibility of the work that we publish. This form provides structure for consistency and transparency in reporting. For further information on Nature Research policies, see Authors \& Referees and the Editorial Policy Checklist.

\section{Statistical parameters}

When statistical analyses are reported, confirm that the following items are present in the relevant location (e.g. figure legend, table legend, main text, or Methods section).

$\mathrm{n} / \mathrm{a} \mid$ Confirmed

$\bigotimes \square$ The exact sample size $(n)$ for each experimental group/condition, given as a discrete number and unit of measurement

\ $\square$ An indication of whether measurements were taken from distinct samples or whether the same sample was measured repeatedly

Х The statistical test(s) used AND whether they are one- or two-sided

Only common tests should be described solely by name; describe more complex techniques in the Methods section.

Х $\square$ A description of all covariates tested

$\bigotimes \square$ A description of any assumptions or corrections, such as tests of normality and adjustment for multiple comparisons

Х $\square$ A full description of the statistics including central tendency (e.g. means) or other basic estimates (e.g. regression coefficient) AND

$\bigotimes$ variation (e.g. standard deviation) or associated estimates of uncertainty (e.g. confidence intervals)

$\bigotimes \square$ For null hypothesis testing, the test statistic (e.g. $F, t, r$ ) with confidence intervals, effect sizes, degrees of freedom and $P$ value noted

$\triangle$ Give $P$ values as exact values whenever suitable.

$\bigotimes \square$ For Bayesian analysis, information on the choice of priors and Markov chain Monte Carlo settings

\ $\square$ For hierarchical and complex designs, identification of the appropriate level for tests and full reporting of outcomes

$\bigotimes \square$ Estimates of effect sizes (e.g. Cohen's $d$, Pearson's $r$ ), indicating how they were calculated

$\bigotimes \square$ Clearly defined error bars

$\triangle \square$ State explicitly what error bars represent (e.g. SD, SE, CI)

Our web collection on statistics for biologists may be useful.

\section{Software and code}

Policy information about availability of computer code

Data collection N/A

Data analysis

N/A

For manuscripts utilizing custom algorithms or software that are central to the research but not yet described in published literature, software must be made available to editors/reviewers upon request. We strongly encourage code deposition in a community repository (e.g. GitHub). See the Nature Research guidelines for submitting code \& software for further information.

\section{Data}

Policy information about availability of data

All manuscripts must include a data availability statement. This statement should provide the following information, where applicable:

- Accession codes, unique identifiers, or web links for publicly available datasets

- A list of figures that have associated raw data

- A description of any restrictions on data availability 


\section{Field-specific reporting}

Please select the best fit for your research. If you are not sure, read the appropriate sections before making your selection.

$\bigotimes$ Life sciences $\quad \square$ Behavioural \& social sciences

For a reference copy of the document with all sections, see nature.com/authors/policies/Reportingsummary-flat.pdf

\section{Life sciences}

\section{Study design}

All studies must disclose on these points even when the disclosure is negative.

Sample size One microinjection session per gRNA. No statistical methods were used.
Data exclusions No data excluded
Replication Nor 100 zygotes were injected for each gRNA.
Randomization Not relevant.
Blinding Not relevant.

\section{Materials \& experimental systems}

Policy information about availability of materials

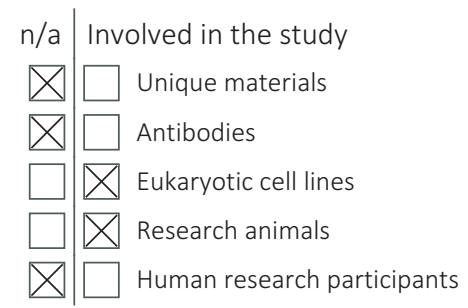

Eukaryotic cell lines

Policy information about cell lines

Cell line source(s)

mouse blastocyst, R1 mouse ES cells

Authentication

blastocyst injection (but not part of this study). R1 was authenticated by generating chimera mice

Mycoplasma contamination

PCR-negative

Commonly misidentified lines

(See ICLAC register)

N/A

Research animals

Policy information about studies involving animals; ARRIVE guidelines recommended for reporting animal research

Animals/animal-derived materials Mus musculus, C57B16, adult.

\section{Method-specific reporting}

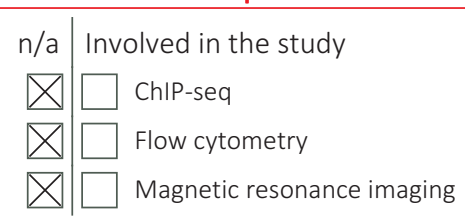




\section{Ma et al. reply}

REPLYING To D. Egli et al. Nature 560, https://doi.org/10.1038/s41586-018-0379-5 (2018); F. Adikusuma et al. Nature 560, https://doi. org/10.1038/s41586-018-0380-z (2018)

The two accompanying Comments ${ }^{1,2}$ highlight the results of our recent study ${ }^{3}$ and their implications for understanding the basic biology of DNA repair and for potential future therapies. Given the importance of the conclusions of our study ${ }^{3}$, further verification was requested to rule out alternative interpretations. One argument made by Egli et $\mathrm{al}^{2}$ was that the repair of the mutant paternal allele using maternalhomologous sequences is highly unlikely based on the assumption that in early zygotes, parental genomes are physically separated in paternal and maternal pronuclei. This temporary isolation would therefore preclude homologous chromosome interactions required for homologydirected repair (HDR). However, it should be noted that CRISPRCas9 ribonucleoprotein (RNP) specific to the mutant paternal allele was delivered into pronuclear-stage zygotes or even earlier during fertilization in our original study ${ }^{3}$, while the subsequent readouts of targeting and repair outcomes were measured three days later in multicellular embryos ${ }^{3}$. In late mammalian zygotes, paternal and maternal pronuclei migrate towards each other with subsequent nuclear envelope breakdown and formation of a diploid mitotic spindle ${ }^{4,5}$. Thus, from this point onwards, parental homologues are presented with ample opportunities to physically interact and recombine. Although we do not exclude the possibility of initial targeting and induction of double-strand breaks (DSBs) in metaphase II (MII) oocytes or zygotes at the time of CRISPR-Cas9 injections, HDR or non-homologous end-joining (NHEJ) could have occurred later during subsequent three mitotic cell cycles. Indeed, we showed that each mosaic 4-8-cell embryo contained blastomeres with two or more different repair outcomes suggesting that CRISPR-Cas9 remains active well beyond the pronuclear stage.

On the basis of the assumption that HDR in pronuclear stage zygotes is impossible, Egli et al. ${ }^{2}$ and Adikusuma et al. ${ }^{1}$ suggested that our results could also represent 'loss' in the detection of the mutant paternal allele altogether owing to large deletions. Such genetic lesions could prevent PCR primer binding and amplification, thus subsequently escaping detection. Adikusuma et al. ${ }^{1}$ indicated the possibility of CRISPR-Cas9 inducing large deletions (greater than 100 base pairs (bp)) at the targeted region, particularly, when disrupting both parental alleles simultaneously. Previous mouse studies showed that the most frequent deletions induced by a single single-guide RNA (sgRNA) do not exceed $10 \mathrm{bp}$ and occurrence of larger deletions are rare to account for the high rates of HDR (above 50\%) observed in our experiments ${ }^{6}$. We designed and pre-tested different sgRNAs in patient induced pluripotent stem cells (iPSCs) and selected one with high specificity for the mutant sequence with no evidence of large deletions detected, making it unlikely, in our view, that our selected sgRNA would induce a large deletion at the frequency we observed for HDR. A recent report also demonstrated that pre-testing of several candidate sgRNAs in human embryonic stem (ES) cells could be effective in predicting editing efficacy of disrupting both copies of POU5F1 in human embryos ${ }^{7}$. In this study, the most frequently observed on-target editing in CRISPR-Cas9microinjected human embryos were small (2-3 bp) indels. Only one embryo contained a few blastomeres with uncommonly large 330-bp deletions ${ }^{7}$.

Although species differences may have impacted editing outcomes, Adikusuma et al. ${ }^{1}$ did not report pre-testing candidate sgRNAs.
Moreover, we used CRISPR-Cas9 RNP, whereas Adikusuma et al. ${ }^{1}$ used Cas 9 mRNA, which may have accounted for large deletions.

Nevertheless, to rule out the possibility of large deletions, we decided to carry out a large-scale re-testing of all embryonic blastomere samples from our published study. Originally, we used PCR amplification followed by Sanger sequencing of a 534-bp fragment spanning approximately $250 \mathrm{bp}$ in each direction from the MYBPC $3^{\triangle G A G T}$ mutation site. To detect larger deletions, we designed an additional 8 pairs of long-range PCR primers amplifying various lengths of fragments surrounding the $M Y B P C 3^{\triangle G A G T}$ mutation locus ranging from $493 \mathrm{bp}$ to 10,160 bp (PCR1-PCR8 in Fig. 1a; Extended Data Table 1). First, we re-tested 8 blastomeres with wild-type maternal and paternal genotypes (WT/WT) from the 4 mosaic embryos S-phase-injected with CRISPR-Cas9 (Extended Data Table 2), along with $4 \mathrm{WT} / \mathrm{WT}$ and 4 wild-type maternal and mutant paternal (WT/Mut) blastomeres from the non-injected control embryos (Extended Data Table 3). PCR products were separated on $1 \%$ agarose gels. In all 16 samples, primers PCR1, PCR2, PCR4 and PCR5 amplified a single band of the expected size (Fig. 1b-e). For PCR6 and PCR7, several faint bands of smaller size were also detectable in some corrected and control blastomeres (Fig. 1f, g). However, Sanger sequencing of these faint bands did not produce any readable products suggesting non-specific PCR primer binding. Next, we performed 2 additional long-range PCR amplifications with PCR3 and PCR8 primers on all remaining WT/WT blastomeres $(n=35)$ from the 13 mosaic embryos along with controls (Extended Data Table 2 and 3). Amplification with PCR3 produced a single band of the expected 1,742 bp size in all experimental and control blastomeres (Fig. 1h). For PCR8, in addition to a major band matching the expected 10,160 bp size, a few faint smaller size bands were also visible in some targeted and control samples, but again, Sanger sequencing indicated non-specific primer binding (Fig. 1i).

We next screened for larger deletions in the M-phase-injected embryos and randomly selected one blastomere from every WT/WT embryo $(n=41)$ as all individual blastomeres within each embryo in this group carried identical MYBPC3 genotypes. We also tested the only mosaic embryo (M2-WT42) in this group that contained 3 blastomeres with WT/WT genotypes and 4 blastomeres with WT/ssODN (Extended Data Table 4). Again, long-range PCR screening of all samples with primers PCR3 and PCR8 produced a single band of expected $1,742 \mathrm{bp}$ or $10,160 \mathrm{bp}$ size, that is, failing to detect large deletions (Fig. 1j, k).

We also examined whole-exome sequencing (WES) results for large deletion in the 6 human ES cell lines derived from M-phase-injected embryos. Comparisons of the area $5 \mathrm{~kb}$ downstream and $5 \mathrm{~kb}$ upstream from the mutation site in ES cells and the corresponding egg and sperm donors revealed no differences in sequencing depth, consistent with the absence of any large deletions (Extended Data Fig. 1).

We then designed two additional $16-\mathrm{kb}$ and $20-\mathrm{kb}$ PCR primers in an attempt to screen for even larger deletions; however, these primers failed to produce a detectable response. All DNA samples were extracted from single blastomeres and then pre-amplified by whole-genome amplification. It is likely that whole-genome amplification results in smaller size DNA segments not compatible for amplification with $16-\mathrm{kb}$ and $20-\mathrm{kb}$ PCR. 


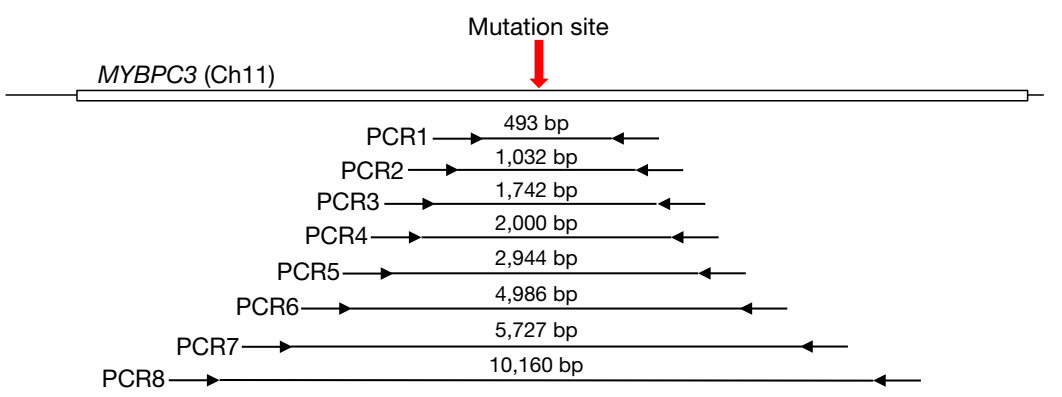

b

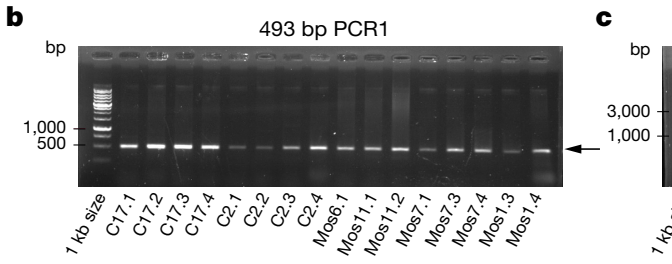

d

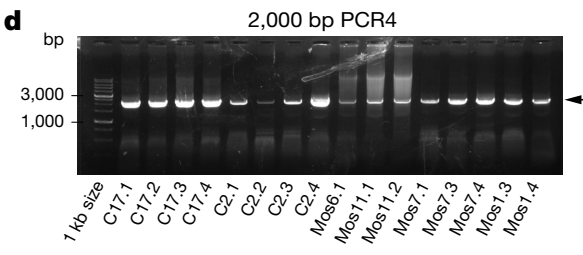

$\mathbf{f}$
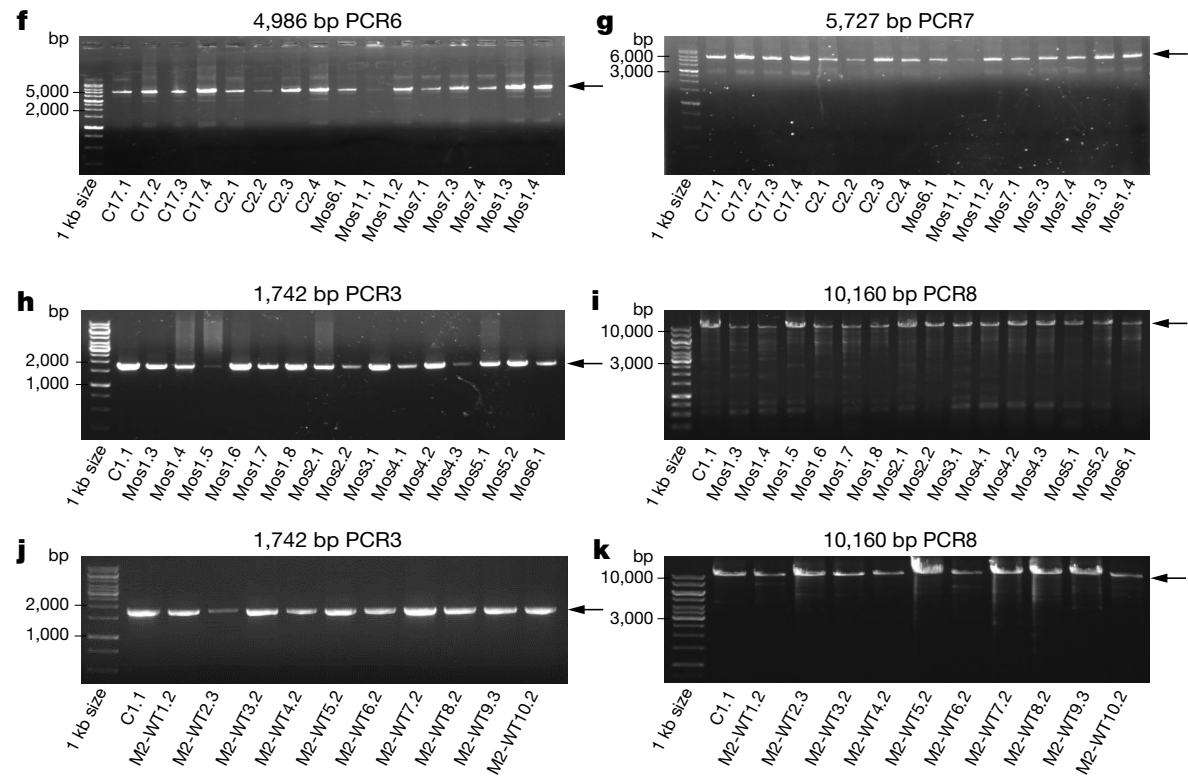

Fig. 1 | Long-range PCR analysis for detection of large deletions in individual blastomeres of mosaic and $\mathrm{M}$-phase-injected human embryos. a, Schematic of eight long-range PCR primers spanning the MYBPC3 ${ }^{\triangle G A G T}$ mutation site. b-g, Agarose gel images of PCR1, PCR2, PCR4-PCR7 amplifications in CRISPR-Cas9-targeted $(n=8)$ and control blastomeres $(n=8)$. $\mathbf{h}, \mathbf{i}$, Representative agarose gel images of PCR3 and PCR8 in

In summary, all these tests failed to detect the presence of large deletions up to $\pm 5 \mathrm{~kb}$ from the mutation site in CRISPR-Cas9-treated human embryos. Although the PCR primers used in this study did not identify much larger deletions, available evidence suggests that most deletions induced by CRISPR-Cas9 should have been detected with our assays. The use of multiple sgRNAs targeting several sites may produce large deletions of up to 24-kb DNA segments; however, the use of single sgRNA has resulted in smaller deletions of less than 600-bp DNA in mouse embryos ${ }^{6}$.
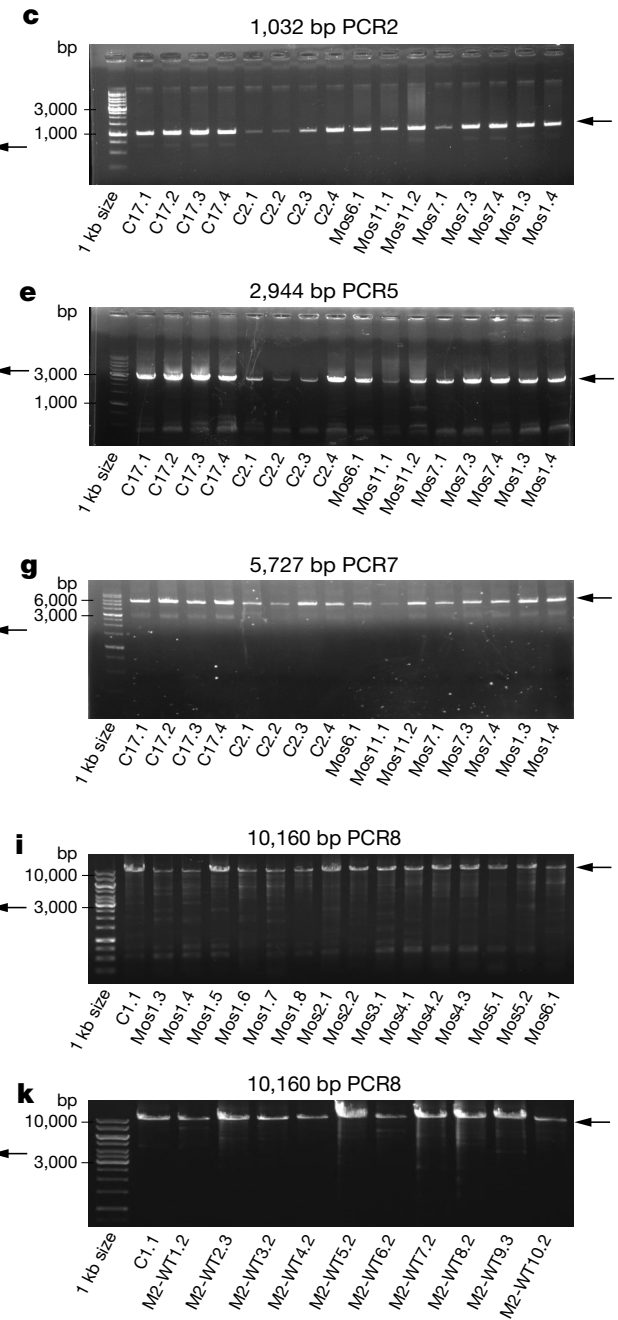

CRISPR-Cas9-targeted ( $n=35)$ and control blastomeres $(n=1)$. j, $\mathbf{k}$, Representative agarose gel images of PCR3 and PCR8 in M-phase-injected WT/WT $(n=48)$ and control blastomeres $(n=1)$. Arrows denote PCR bands reflecting the expected DNA size. Similar results were obtained from two independent PCR amplifications for each sample presented in $\mathbf{b}-\mathbf{k}$.

Our original results suggest that DSB repair on the paternal allele governed by maternal homologue-based HDR extends to the adjacent $\triangle$ GAGT deletion site resulting in conversion of the paternal sequence (see extended data figure $2 \mathrm{a}$ of the original study ${ }^{3}$ ). Therefore, we asked whether DNA proofreading and mismatch repair mechanisms involved in HDR could also contribute to the conversion of neighbouring neutral paternal SNPs resulting in loss-of-heterozygosity $(\mathrm{LOH})$ within the MYBPC3 locus. We postulated that paternal single nucleotide polymorphisms (SNPs) adjacent to the targeted DSB locus would be 


\section{BRIEF COMMUNICATIONS ARISING}

a
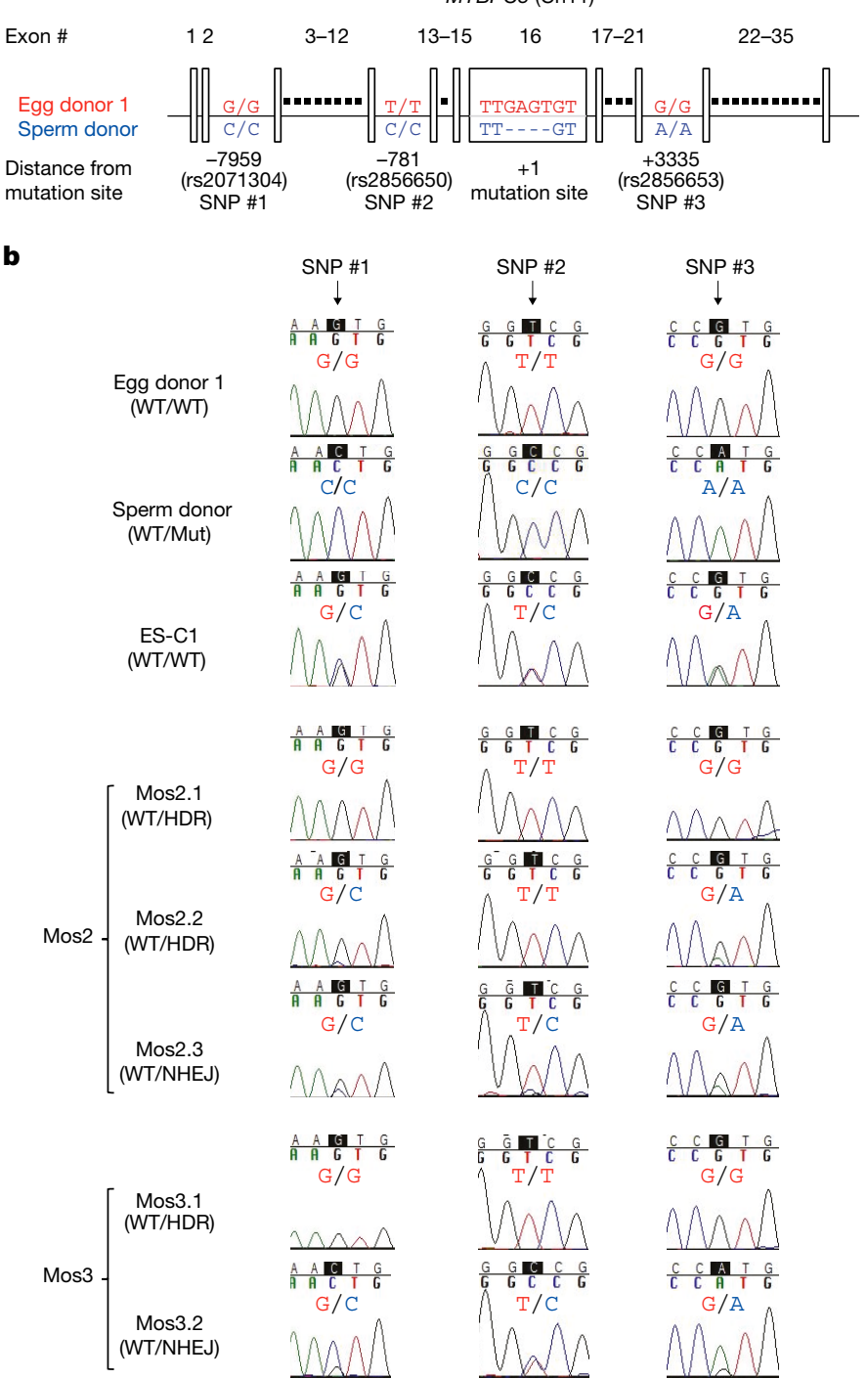

Fig. 2 Evaluation of HDR repair and conversion tract length in mosaic embryos and control embryonic stem cells produced from egg donor 1 . a, Schematic map of three informative SNPs within a genomic region of the MYBPC3 gene. The rs number under each SNP represents a reference number recorded at NCBI dbSNP (the Short Genetic Variation database). b, Representative chromatographs of SNP genotypes in individual blastomeres $(n=5)$ from mosaic embryos and control embryonic stem cells. Similar results were obtained from two independent Sanger sequencing for each sample presented in $\mathbf{b}$.

converted to become maternal-like, while more distant polymorphic sites would be preserved. We searched WES and whole-genome sequencing (WGS) datasets and identified three informative parental SNPs within the MYBPC3 gene distinguishing egg donor 1 from the sperm donor. SNPs \#1 (rs2071304) and \#2 (rs2856650) were located downstream of the $\Delta$ GAGT deletion site ( $-7,959 \mathrm{bp}$ and $-781 \mathrm{bp}$ ), whereas SNP \#3 (rs2856653) was $+3,335$ bp upstream from this locus (Fig. 2a). We next genotyped individual blastomeres of the two CRISPR-Cas9-injected mosaic embryos (Mos2 and Mos3 in Table 1) from this parental combination. The ES cell line (ES-C1) derived from the control non-injected, blastocyst from the same parental combination was also genotyped. ES-C1 with the WT/WT genotype at the mutation locus and two blastomeres, Mos2.3 and Mos3.2, from the mosaic embryos with the WT/NHEJ genotype were heterozygous at all three polymorphic sites representing the expected maternal and paternal SNPs (G/C, T/C and G/A for SNPs \#1, \#2 and \#3, respectively) (Table 1 and Fig. 2b). By contrast, the two blastomeres Mos2.1 and Mos3.1 with the WT/HDR genotypes from the same mosaic embryos were homozygous for all three SNP sites carrying exclusively maternal nucleotides. Notably, another blastomere, Mos2.2, also with a WT/HDR genotype, was homozygous at the SNP \#2 locus carrying maternal nucleotides, but heterozygous at both SNP \# 1 (G/C) and SNP \#3 (G/A), indicating preservation of these paternal SNP sites (Table 1 and Fig. 2b). These results support the notion that HDR-based conversion can expand beyond the targeted mutant loci, resulting in loss of neutral paternal SNPs across the MYBPC3 region. However, the acquisition of maternal SNPs by something other than long conversion tract cannot be completely ruled out.

In contrast to the mosaic counterparts, the MYBPC3 genotype of the original sperm in uniform WT/WT embryos produced from CRISPRCas9-treated zygotes or oocytes cannot be determined. Nevertheless, we suggested that some embryos with $M Y B P C 3^{W T / W T}$ genotypes could have originated from mutant $M Y B P C 3^{\triangle G A G T}$ sperm, with subsequent HDR correction of the deletion. This original assumption was based on a significant increase in the percentage of WT/WT embryos in the CRISPR-Cas9-treated group compared to non-treated controls ${ }^{3}$. We reasoned that loss of neutral paternal SNPs in some of these WT/WT embryos could be used as evidence of repair of the mutant $M Y B P C 3^{\triangle G A G T}$. Among $42 \mathrm{WT} / \mathrm{WT}$, M-phase-injected embryos, six (M2-WT28 to M2-WT33 in Table 1) were derived from the egg donor 1 and the sperm donor, and thus should be heterozygous at the SNP \#1, $\# 2$ and \#3 sites. We randomly genotyped two sister blastomeres from each of these six embryos, and documented $\mathrm{LOH}$ in at least one of these polymorphic sites in four embryos. As expected, paternal SNPs were lost at these loci, resulting in homozygous maternal nucleotides (Table 1 and Fig. 3). Notably, genotypes of two sister blastomeres from the same embryo were distinct from each other, suggesting independent HDR events probably occurred at the two-cell stage or later. For example, one blastomere (M2-WT29.3) in embryo M2-WT29 was homozygous at all three SNP loci carrying exclusively maternal nucleotides while the other sister blastomere (M2-WT29.2) was heterozygous at all three SNP sites (Table 1 and Fig. 3). A similar pattern was also observed in embryos M2-WT 30 and 32. By contrast, one blastomere (M2-WT31.1) of embryo M2-WT31 was homozygous containing maternal alleles at the SNP \#2 and \#3 (T/T and G/G, respectively), whereas the more distant SNP\#1 was heterozygous (G/C). Its sister blastomere M2-WT31.2 was heterozygous at these three SNP positions.

As indicated above, $\mathrm{LOH}$ associated with erasure of paternal SNPs in these four uniform WT/WT embryos provides support for repair of the mutant sperm MYBPC $3^{\triangle G A G T}$ deletion following CRISPR-Cas9 treatment. Additional genotyping of distant SNPs throughout chromosome 11 from all parental contributions would be necessary to establish the median conversion tract length. All examined blastomeres in the remaining embryos, M2-WT28 and M2-WT33, were heterozygous at all three SNP sites, suggesting that these embryos were fertilized by wild-type sperm.

We extended SNP analysis to four uniform WT/WT embryos from the S-phase-injected group from the same parental combination. Three embryos (WT4, WT5 and WT6) were heterozygous for all three SNPs, whereas both blastomeres examined from WT3 embryo were heterozygous at SNP \#1 and \#3 but homozygous at SNP \#2 (Table 1 and Fig. 3). Thus, this embryo was probably generated from the mutant sperm but subsequently corrected by HDR using the wild-type maternal allele.

To provide further genetic evidence for HDR, we also screened egg donor 2 and identified two informative SNPs within the MYBPC3 gene that would differentiate from the paternal contribution. Egg donor 2 was homozygous (G/G) at the SNP \#4 site (positioned -6,189 bp downstream of the $\triangle$ GAGT mutation, rs2697920), whereas the sperm 
Table 1 | MYBPC3 SNP genotypes in embryos from egg donor 1 and $M Y B P C 3^{\triangle G A G T}$ sperm donor

\begin{tabular}{|c|c|c|c|c|c|c|}
\hline Treatment & Samples & Blastomere ID & MYBPC3 genotype & SNP \#1 -7959a & SNP\#2 $-781^{a}$ & $\mathrm{SNP} \# 3+3335^{\mathrm{a}}$ \\
\hline & Egg donor 1 & $\mathrm{~N} / \mathrm{A}$ & WT/WT & $\mathbf{G} / \mathbf{G}$ & $\mathbf{T} / \mathbf{T}$ & $\mathbf{G} / \mathbf{G}$ \\
\hline & Sperm donor & $N / A$ & WT/Mut & $\mathrm{C} / \mathrm{C}$ & $\mathrm{C} / \mathrm{C}$ & $A / A$ \\
\hline Control & ES-C1 & $\mathrm{N} / \mathrm{A}$ & WT/WT & $\mathbf{G} / \mathrm{C}$ & $\mathbf{T} / \mathrm{C}$ & $\mathbf{G} / \mathrm{A}$ \\
\hline \multirow[t]{5}{*}{ S-phase-injected } & Mos2 & Mos2.3 & WT/NHEJ & $\mathbf{G} / \mathrm{C}$ & $\mathbf{T} / \mathrm{C}$ & $\mathbf{G} / \mathrm{A}$ \\
\hline & & Mos2.1 & WT/HDR & $\mathbf{G} / \mathbf{G}$ & $\mathbf{T} / \mathbf{T}$ & $\mathbf{G} / \mathbf{G}$ \\
\hline & & Mos2.2 & WT/HDR & $\mathbf{G} / C$ & $\mathbf{T} / \mathbf{T}$ & $\mathbf{G} / \mathrm{A}$ \\
\hline & Mos3 & Mos3.2 & WT/NHEJ & $\mathbf{G} / \mathrm{C}$ & $\mathbf{T} / \mathrm{C}$ & $\mathbf{G} / \mathbf{A}$ \\
\hline & & Mos3.1 & WT/HDR & $\mathbf{G} / \mathbf{G}$ & $\mathbf{T} / \mathbf{T}$ & $\mathbf{G} / \mathbf{G}$ \\
\hline \multirow[t]{8}{*}{ S-phase-injected } & WT3 & WT3.3 & WT/WT & $\mathbf{G} / \mathrm{C}$ & $\mathbf{T} / \mathbf{T}$ & $\mathbf{G} / \mathrm{A}$ \\
\hline & & WT3.4 & WT/WT & $\mathbf{G} / \mathrm{C}$ & $\mathbf{T} / \mathbf{T}$ & $\mathbf{G} / \mathrm{A}$ \\
\hline & WT4 & WT4.1 & WT/WT & $\mathbf{G} / \mathrm{C}$ & $\mathrm{T} / \mathrm{C}$ & $\mathbf{G} / \mathrm{A}$ \\
\hline & & WT4.4 & WT/WT & $\mathbf{G} / \mathrm{C}$ & $\mathbf{T} / \mathrm{C}$ & $\mathbf{G} / \mathrm{A}$ \\
\hline & WT5 & WT5.1 & WT/WT & $\mathbf{G} / \mathrm{C}$ & $\mathrm{T} / \mathrm{C}$ & $\mathbf{G} / \mathrm{A}$ \\
\hline & & WT5.2 & WT/WT & $\mathbf{G} / \mathrm{C}$ & $\mathrm{T} / \mathrm{C}$ & $\mathbf{G} / \mathrm{A}$ \\
\hline & WT6 & WT6.1 & WT/WT & $\mathbf{G} / \mathrm{C}$ & $\mathrm{T} / \mathrm{C}$ & $\mathbf{G} / \mathrm{A}$ \\
\hline & & WT6.2 & WT/WT & $\mathbf{G} / \mathrm{C}$ & $\mathrm{T} / \mathrm{C}$ & $\mathbf{G} / \mathrm{A}$ \\
\hline \multirow[t]{12}{*}{ M-phase-injected } & M2-WT29 & M2-WT29.2 & WT/WT & $\mathbf{G} / \mathrm{C}$ & $\mathbf{T} / \mathrm{C}$ & $\mathbf{G} / \mathbf{A}$ \\
\hline & & M2-WT29.3 & WT/WT & $\mathbf{G} / \mathbf{G}$ & $\mathbf{T} / \mathbf{T}$ & $\mathrm{G} / \mathrm{G}$ \\
\hline & M2-WT30 & M2-WT30.2 & WT/WT & $\mathbf{G} / \mathbf{G}$ & $\mathbf{T} / \mathbf{T}$ & $\mathbf{G} / \mathbf{G}$ \\
\hline & & M2-WT30.3 & WT/WT & $\mathbf{G} / \mathrm{C}$ & $\mathrm{T} / \mathrm{C}$ & $\mathbf{G} / \mathrm{A}$ \\
\hline & M2-WT31 & M2-WT31.1 & WT/WT & $\mathbf{G} / \mathrm{C}$ & $\mathbf{T} / \mathbf{T}$ & $\mathbf{G} / \mathbf{G}$ \\
\hline & & M2-WT31.2 & WT/WT & $\mathbf{G} / \mathrm{C}$ & $\mathbf{T} / \mathrm{C}$ & $\mathbf{G} / \mathrm{A}$ \\
\hline & M2-WT32 & M2-WT32.2 & WT/WT & $\mathbf{G} / \mathrm{C}$ & $\mathrm{T} / \mathrm{C}$ & $\mathbf{G} / \mathrm{A}$ \\
\hline & & M2-WT32.3 & WT/WT & $\mathbf{G} / \mathbf{G}$ & $\mathbf{T} / \mathbf{T}$ & $\mathbf{G} / \mathbf{G}$ \\
\hline & M2-WT28 & M2-WT28.1 & WT/WT & $\mathbf{G} / \mathrm{C}$ & $\mathbf{T} / \mathrm{C}$ & $\mathbf{G} / \mathrm{A}$ \\
\hline & & M2-WT28.5 & WT/WT & $\mathbf{G} / \mathrm{C}$ & $\mathbf{T} / \mathrm{C}$ & $\mathbf{G} / \mathrm{A}$ \\
\hline & M2-WT33 & M2-WT33.2 & WT/WT & $\mathbf{G} / \mathrm{C}$ & $\mathbf{T} / \mathrm{C}$ & $\mathbf{G} / \mathrm{A}$ \\
\hline & & M2-WT33.3 & WT/WT & $\mathbf{G} / \mathrm{C}$ & $\mathrm{T} / \mathrm{C}$ & $\mathbf{G} / \mathrm{A}$ \\
\hline
\end{tabular}

MYBPC3 SNP genotypes in individual blastomeres of S-phase- and M-phase-injected embryos derived from egg donor 1 and the MYBPC ${ }^{\triangle G A G T}$ mutant sperm donor. Bold font indicates maternal nucleotides; non-bold font represents paternal nucleotides

$\mathrm{N} / \mathrm{A}$, not applicable.

aDownstream and upstream distance from the 4-bp deletion in the MYBPC3 gene.

donor was heterozygous (A/G) at this locus (Extended Data Fig. 2a). At SNP \#5 (+9,514 bp, rs11570115), both parents were heterozygous A/G. We initially genotyped blastomeres with WT/NHEJ or WT/Mut genotypes from seven mosaic embryos (Mos1, Mos7, Mos8, Mos10, Mos11, Mos12, and Mos13) derived from this parental combination, and found that six were heterozygous A/G at the SNP \#4 locus (Table 2, footnote symbol b), indicating that mutant sperm contributed the ' $\mathrm{A}$ ' allele at this locus in these embryos. We next sequenced all sister blastomeres with WT/HDR genotypes from these six embryos and found that five (Mos1, Mos7, Mos8, Mos10 and Mos13) contained one or more blastomeres that lost the paternal allele and became homozygous G/G at SNP \#4, supporting the gene conversion from maternal allele (Table 2 and Extended Data Fig. 2b). The remaining WT/HDR blastomeres in these embryos retained the paternal allele and were heterozygous $\mathrm{A} / \mathrm{G}$ at the SNP\#4, probably indicating a shorter conversion tract. Both WT/HDR blastomeres from Mos11 embryo were heterozygous A/G at SNP \#4. WT/Mut blastomere from Mos12 embryo was homozygous $\mathrm{G} / \mathrm{G}$ at SNP \#4, precluding the need for further genotyping determinations (Table 2).

We next sequenced one randomly selected blastomere from each of the seven uniform WT/WT, M-phase-injected embryos generated from the egg donor 2 and the sperm donor. We found all seven blastomeres were homozygous G/G at SNP \#4 (Table 2). In comparison, six out of seven S-phase-injected mosaic embryos generated from the same parental combination were heterozygous A/G (Table 2). Therefore, we think it is possible that some of these G/G homozygous embryos in the M-phase-injected group also lost paternal SNPs owing to gene conversion. Genotyping for the SNP \#5 locus showed that two mosaic S-phase-injected embryos (Mos1 and Mos8) were heterozygous $\mathrm{A} / \mathrm{G}$ and informative for conversion analyses (Table 2). All five sister blastomeres with WT/HDR genotypes in the Mos1 embryo were homozygous G/G at SNP \#5, indicating loss of paternal SNPs. Of the two WT/HDR blastomeres in Mos8 embryo, one was homozygous G/G and one was heterozygous A/G at SNP \#5. Among M-phase-injected embryos, one out of seven was heterozygous $A / G$ and the remaining six were homozygous G/G at SNP \#5 (Table 2).

Together, these results suggest that gene conversion in human embryos induced by HDR may happen and extend considerable distances in both directions from the original target site, resulting in $\mathrm{LOH}$ associated with erasure of neutral paternal SNPs. The length of the conversion tract varied among individual blastomeres even from the same embryo. The existence of polymorphic sites and retention of paternal SNPs on some corrected blastomeres also provides a strong suggestion that the mutant paternal MYPBC3 locus was repaired in embryos injected at the $S$ phase and $M$ phase.

In our original study ${ }^{3}$, we demonstrated that early exposure to CRISPR-Cas9 RNP during fertilization (M phase) could considerably reduce or completely eliminate mosaicism in cleaving embryos. 


\section{BRIEF COMMUNICATIONS ARISING}

MYBPC3 (Ch11)

\begin{tabular}{llccc} 
Egg donor 1 & $\mathrm{G} / \mathrm{G}$ & $\mathrm{T} / \mathrm{T}$ & $\mathrm{G} / \mathrm{G}$ \\
\cline { 2 - 5 } Sperm donor & $\mathrm{C} / \mathrm{C}$ & $\mathrm{C} / \mathrm{C}$ & $\uparrow$ & $\mathrm{A} / \mathrm{A}$ \\
Distance from & -7959 & -781 & +1 & +3335 \\
mutation site & SNP \#1 & SNP \#2 & mutation & SNP \#3
\end{tabular}

site

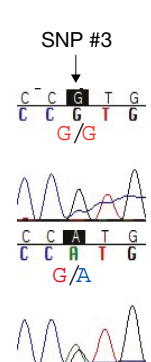

M2-WT29.2

M2-WT29.3

(WT/WT)

M2-WT29
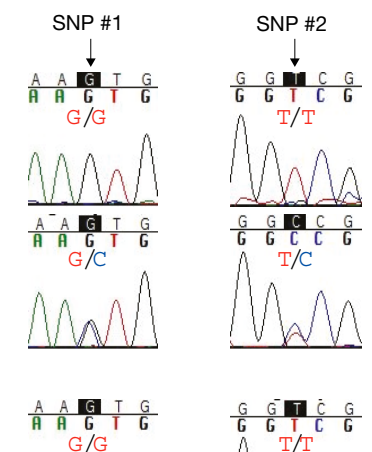

M2-WT30.2

(WT/WT)

M2-WT30

M2-WT30.3

(WT/WT)
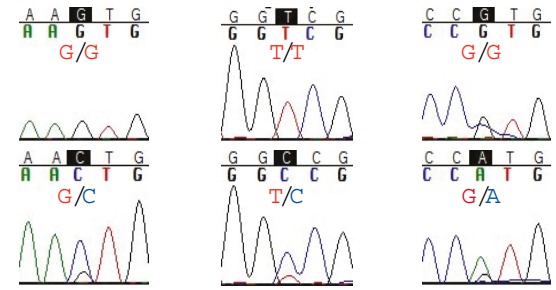

M2-WT31.1 (WT/WT) M2-WT31.2 (WT/WT)
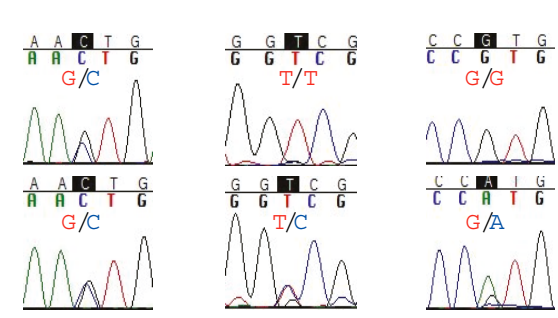

$\mathrm{G} / \mathrm{A}$
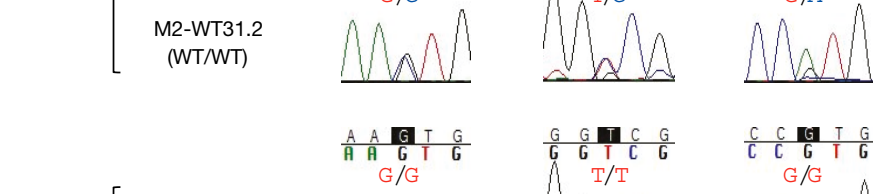
$\left[\begin{array}{c}\text { M2-WT32.3 } \\ \text { (WT/WT) }\end{array}\right.$

M2-WT32

M2-WT32.2

(WT/WT)

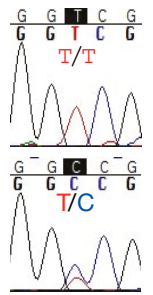

(figure 2a of the original study ${ }^{3}$ ). Egli et al. ${ }^{2}$ argue that the increased yield of WT/WT embryos (22\%) in the M-phase-injected group could also be due to parthenogenetic development. Of $75 \mathrm{M}$-phase-injected oocytes, 2 were lysed during intracytoplasmic sperm injection and 10 failed to fertilize. The remaining 63 (84\%) exhibited normal fertilization morphology with two pronuclei and two polar bodies, inconsistent with parthenogenic activation. Similar results were obtained from non-injected controls and S-phase-injected embryos (extended data table 2 of the original study ${ }^{3}$ ). Moreover, SNP analyses provided in Tables 1 and 2 for WT/WT embryos in the M-phase-injected group clearly demonstrate retention of paternal SNPs. To further exclude the possibility of parthenogenetic development, we confirmed the paternal contribution in WT/WT ES cell lines derived from M-phase-injected embryos by short tandem repeat (STR) assay. As expected, all six ES cell lines derived by M-phase injection and one non-injected control (extended data figure $3 \mathrm{a}$ of the original study ${ }^{3}$ ) contained both maternal and paternal STR alleles (Extended Data Table 5). Thus, in all samples we analysed, paternal contribution was detected and parthenogenesis could be ruled out.

Mounting evidence suggests that the two parental homologues provide more than a genetic diversity contributed by parents ${ }^{8}$. Recent developments in custom-designed nucleases allowing selective targeting of one of the two parental alleles have provided evidence for inter-chromosomal pairing, interaction and contribution to DNA repair across plant and animal species. Among the possible interactions are DNA DSB repair governed by mitotic recombination or homologue-template-based repair contributing to $\mathrm{LOH}^{9}$. A more recent study using mutant tomato plants with different fruit colours concluded that in heterozygous plants, CRISPR-Cas9-induced DSBs in the targeted allele were repaired using the intact allele as a template at a frequency up to $14 \%$ and that HDR between homologues occurred in the absence of the meiotic machinery ${ }^{10}$. Specific targeting of the mutant paternal allele in heterozygous mice also demonstrated that DSB repair, via HDR using the wild-type maternal allele, resulted in the birth of viable WT/WT offspring ${ }^{11}$. DSB induction in both parental alleles simultaneously could also induce template-mediated repair using endogenous genomic sequences from close homologous gene families. In human zygotes, CRISPR-Cas9 based bi-allelic targeting of the $\beta$-globin gene $(H B B)$ resulted in HDR using the endogenous $\delta$-globin gene $(H B D)^{12}$.

On the basis of our original results ${ }^{3}$ and those present here, we suggest that human embryos have the capacity for non-meiotic homologous chromosome-based DNA repair. This endogenous repair competence must be further explored, reproduced with different founder mutations, and perhaps evaluated for future germline gene therapeutic applications. Many questions remain concerning the precise mechanisms involved during homologous chromosome-based HDR and cell cycle timing. However, given that such DNA repair seems widely conserved across different species, in depth mechanistic studies can potentially be addressed in model organisms. Indeed, a recent article in bioRxiv confirmed our findings in the mouse using more rigorous analyses ${ }^{13}$. Wilde et al. ${ }^{13}$ validated high frequency of inter-homologue HDR mechanism in mouse heterozygous zygotes and demonstrated a significant increase of this process by complementing with the HDR-associated strand exchange factor RAD51. In the interim, we hope that the questions raised by Egli et al. ${ }^{2}$ and Adikusuma et al. ${ }^{1}$ and our new results presented here will contribute to a better understanding of the complex nature of DNA repair and serve as a useful platform for further discussions and studies.

\section{Methods}

Regulations for studies involving human research participants. Oregon Health \& Science University (OHSU) has established a strong regulatory framework that allowed for approval and continued monitoring of this study protocol. Approval was granted by all relevant regulatory bodies; OHSU Institutional IRB 
Table 2 | MYBPC3 SNP genotypes in embryos from egg donor 2 and $M Y B P C 3^{\triangle G A G T}$ sperm donor

\begin{tabular}{|c|c|c|c|c|c|}
\hline Treatment & Samples & Blastomere ID & MYBPC3 genotype & SNP \#4 -6189a & SNP \#5 $+9514^{a}$ \\
\hline & Egg donor 2 & N/A & WT/WT & $\mathbf{G} / \mathbf{G}$ & $A / G$ \\
\hline & Sperm donor & $\mathrm{N} / \mathrm{A}$ & WT/Mut & $A / G$ & $A / G$ \\
\hline \multirow[t]{6}{*}{ S-phase-injected } & Mos1 & Mos1.1 & WT/NHEJ ${ }^{b}$ & $A / \mathbf{G}$ & $A / G$ \\
\hline & & Mos 1.4 & WT/HDR & $\mathrm{G} / \mathrm{G}$ & $\mathrm{G} / \mathrm{G}$ \\
\hline & & Mos1.5 & WT/HDR & $\mathrm{G} / \mathrm{G}$ & $\mathrm{G} / \mathrm{G}$ \\
\hline & & Mos 1.6 & WT/HDR & $A / \mathbf{G}$ & $\mathrm{G} / \mathrm{G}$ \\
\hline & & Mos 1.7 & WT/HDR & $\mathrm{G} / \mathrm{G}$ & $\mathrm{G} / \mathrm{G}$ \\
\hline & & Mos 1.8 & WT/HDR & $A / \mathbf{G}$ & $\mathrm{G} / \mathrm{G}$ \\
\hline \multirow[t]{4}{*}{ S-phase-injected } & Mos7 & Mos7.2 & WT/NHEJ ${ }^{\mathrm{b}}$ & $A / \mathbf{G}$ & $A / A$ \\
\hline & & Mos7.1 & WT/HDR & $A / \mathbf{G}$ & $\mathrm{N} / \mathrm{A}$ \\
\hline & & Mos7.3 & WT/HDR & $\mathrm{G} / \mathrm{G}$ & $\mathrm{N} / \mathrm{A}$ \\
\hline & & Mos7.4 & WT/HDR & $A / \mathbf{G}$ & $\mathrm{N} / \mathrm{A}$ \\
\hline \multirow[t]{3}{*}{ S-phase-injected } & Mos8 & Mos8.3 & WT/Mut ${ }^{b}$ & $A / \mathbf{G}$ & $A / G$ \\
\hline & & Mos8.1 & WT/HDR & $\mathrm{G} / \mathrm{G}$ & $\mathrm{A} / \mathrm{G}$ \\
\hline & & Mos8.2 & WT/HDR & $A / \mathbf{G}$ & $\mathrm{G} / \mathrm{G}$ \\
\hline \multirow[t]{5}{*}{ S-phase-injected } & Mos10 & Mos 10.5 & WT/Mut ${ }^{\mathrm{b}}$ & $A / \mathbf{G}$ & $\mathrm{A} / \mathrm{A}$ \\
\hline & & Mos10.1 & WT/HDR & $\mathrm{G} / \mathrm{G}$ & $\mathrm{N} / \mathrm{A}$ \\
\hline & & Mos10.2 & WT/HDR & $A / \mathbf{G}$ & $N / A$ \\
\hline & & Mos10.3 & WT/HDR & $A / \mathbf{G}$ & $\mathrm{N} / \mathrm{A}$ \\
\hline & & Mos10.4 & WT/HDR & $A / \mathbf{G}$ & $N / A$ \\
\hline \multirow[t]{3}{*}{ S-phase-injected } & Mos11 & Mos11.3 & WT/NHEJb & $A / \mathbf{G}$ & $\mathrm{A} / \mathrm{A}$ \\
\hline & & Mos11.1 & WT/HDR & $A / \mathbf{G}$ & $N / A$ \\
\hline & & Mos11.2 & WT/HDR & $A / \mathbf{G}$ & $\mathrm{N} / \mathrm{A}$ \\
\hline \multirow[t]{2}{*}{ S-phase-injected } & Mos13 & Mos13.3 & WT/Mut ${ }^{\mathrm{b}}$ & $A / \mathbf{G}$ & $A / A$ \\
\hline & & Mos13.1 & WT/HDR & $\mathrm{G} / \mathrm{G}$ & $\mathrm{N} / \mathrm{A}$ \\
\hline \multirow[t]{3}{*}{ S-phase-injected } & Mos12 & Mos12.8 & WT/NHEJ & $\mathrm{G} / \mathrm{G}$ & $\mathrm{G} / \mathrm{G}$ \\
\hline & & Mos12.1 & WT/HDR & $\mathrm{G} / \mathrm{G}$ & $\mathrm{N} / \mathrm{A}$ \\
\hline & & $\operatorname{Mos} 12.2$ & WT/HDR & $\mathrm{G} / \mathrm{G}$ & $\mathrm{N} / \mathrm{A}$ \\
\hline \multirow[t]{7}{*}{ M-phase-injected } & M2-WT1 & M2-WT1.2 & WT/WT & $\mathrm{G} / \mathrm{G}$ & $A / G$ \\
\hline & M2-WT2 & M2-WT2.2 & WT/WT & $\mathrm{G} / \mathrm{G}$ & $\mathrm{G} / \mathrm{G}$ \\
\hline & M2-WT3 & M2-WT3.2 & WT/WT & $\mathrm{G} / \mathrm{G}$ & $\mathrm{G} / \mathrm{G}$ \\
\hline & M2-WT4 & M2-WT4.3 & WT/WT & $\mathrm{G} / \mathrm{G}$ & $\mathrm{G} / \mathrm{G}$ \\
\hline & M2-WT5 & M2-WT5.2 & WT/WT & $\mathrm{G} / \mathrm{G}$ & $\mathrm{G} / \mathrm{G}$ \\
\hline & M2-WT6 & M2-WT6.2 & WT/WT & $\mathrm{G} / \mathrm{G}$ & $\mathrm{G} / \mathrm{G}$ \\
\hline & M2-WT7 & M2-WT7.2 & WT/WT & $\mathrm{G} / \mathrm{G}$ & $\mathrm{G} / \mathrm{G}$ \\
\hline
\end{tabular}

MYBPC3 SNP genotypes in individual blastomeres of S-phase and M-phase-injected embryos derived from egg donor 2 and the MYBPC ${ }^{\triangle G A G T}$ mutant sperm donor. Bold font indicates maternal nucleotides; italic font shows parental nucleotides. N/A, not applicable.

a Represents downstream and upstream distance from the 4 bp deletion in the MYBPC3 gene.

bBlastomeres with WT/Mut or WT/NHEJ MYBPC3 genotypes that were heterozygous A/G at SNP \#4.

(IRB), OHSU Scientific Review Committee (SRC), OHSU Innovative Research Advisory Panel (IRAP), and Data Safety Monitoring Committee (DSMC). These committees are informed by national and international guidelines published by the Hinxton group, the International Society for Stem Cell Research (ISSCR) and the National Academy of Science and Medicine committee reports.

Healthy gamete donors were recruited locally via print and web-based advertising and underwent ovarian stimulation at OHSU. A single sperm donor with a heritable MYBPC3 mutation was identified by physicians from the OHSU Knight Cardiovascular Institute and referred to the research team. Written informed consent was obtained from all participants before study-related procedures. The current study complied with all relevant ethical regulations.

Long-range PCR and Sanger sequencing. Long-range PCR (PCR1, PCR2 and PCR4-PCR7) was performed using PrimeSTAR GXL DNA Polymerase, whereas the long-range PCR3 and PCR8 were performed with TaKaRa LA Taq DNA Polymerase (Clontech) according to manufacturer's procedure. In brief, PCR conditions were $10 \mathrm{~s}$ at $98^{\circ} \mathrm{C}, 15 \mathrm{~s}$ at $60^{\circ} \mathrm{C}$, and $1 \mathrm{~min} \mathrm{~kb}^{-1}$ at $68^{\circ} \mathrm{C}$ (30-35 cycles). PCR products were resolved with $1 \%$ agarose gel electrophoresis and were visualized with EtBr staining.
For Sanger sequencing, targeted region PCR for each single nucleotide polymorphisms (SNPs) was carried out using the PCR Platinum SuperMix High Fidelity Kit (Life Technologies). The PCR products were Sanger sequenced and analysed by Sequencher v5.0 (GeneCodes).

Parentage analysis by STR assay. DNA was extracted from blood of egg and sperm donors and individual ES cell lines using commercial kits (Gentra). STR microsatellite parentage analysis was conducted by the Genetics Laboratory at University of California, Davis as described previously ${ }^{14}$.

SNP searching and calling using WES and WGS. WES sequencing data were first processed by filtering adaptor sequences and removing low quality reads or reads with a high percentage of $\mathrm{N}$ bases using SOAPnuke (1.5.2) software (http://soap.genomics.org.cn/) developed by BGI. Clean reads were generated for each library. Clean data were paired-end aligned using the Burrows-Wheeler Aligner ${ }^{15}$ (BWA) program version 0.7.12 to the human genome assembly hg19. Duplicate reads in alignment BAM files were identified using MarkDuplicates in Picard v1.54 (https://broadinstitute.github.io/picard/). The alignment results were processed by RealignerTargetCreator, IndelRealigner and BaseRecalibrator modules in $\operatorname{GATK}^{16}$ (3.3.0) and variants detection was performed by HaplotypeCaller 
tool in GATK according to GATK Best Practices recommendations ${ }^{17,18}$. SNV and InDel information were extracted and filtered by VQSR in GATK and annotated by AnnoDB v3 (http://www.igm.columbia.edu/resources/bioinformatics). Data availability. The datasets including Sanger sequencing, STR, WES and WGS generated and analysed during this study are not publicly available to protect the identity and privacy of study participants. However, the data will be available from the corresponding author upon individual requests and after OHSU IRB/ DSMC approvals.

Hong $\mathrm{Ma}^{1}$, Nuria Marti-Gutierrez ${ }^{1}$, Sang-Wook Park ${ }^{2}$, Jun $\mathrm{Wu}^{3,11}$, Tomonari Hayama ${ }^{1}$, Hayley Darby ${ }^{1}$, Crystal Van Dyken ${ }^{1}$, Ying $\mathrm{Li}^{1}$, Amy Koski ${ }^{1}$, Dan Liang ${ }^{1}$, Keiichiro Suzuki ${ }^{3}$, Ying $\mathrm{Gu}^{4,5,6}$, Jianhui Gong ${ }^{4,5,6,7}$, Xun Xu ${ }^{4,5,6}$, Riffat Ahmed ${ }^{1}$, Yeonmi Lee ${ }^{1,12}$, Eunju Kang ${ }^{1,12}$, Dongmei Ji ${ }^{1,13}$, A-Reum Park ${ }^{2}$, Daesik Kim ${ }^{8}$, Sang-Tae Kim² ${ }^{2}$, Stephen B. Heitner ${ }^{9}$, David Battaglia ${ }^{1,10}$, Sacha A. Krieg ${ }^{10}$, David M. Lee ${ }^{10}$, Diana H. Wu ${ }^{10}$, Don P. Wolf ${ }^{1}$, Paula Amato ${ }^{1,10}$, Sanjiv Kaul ${ }^{9}$, Juan Carlos Izpisua Belmonte ${ }^{3}$, Jin-Soo Kim ${ }^{2,8}$ \& Shoukhrat Mitalipov ${ }^{1,9,10 *}$

${ }^{1}$ Center for Embryonic Cell and Gene Therapy, Oregon Health \& Science University, Portland, OR, USA. ${ }^{2}$ Center for Genome Engineering, Institute for Basic Science, Daejeon, South Korea. ${ }^{3}$ Gene Expression Laboratory, Salk Institute for Biological Studies, La Jolla, CA, USA. "BGI-Shenzhen, Beishan Industrial Zone, Shenzhen, China. ${ }^{5}$ China National GeneBank, BGl-Shenzhen, Shenzhen, China. ${ }^{6} \mathrm{BGI}$-Qingdao, Qingdao, China. ${ }^{7}$ Shenzhen Engineering Laboratory for Innovative Molecular Diagnostics, BGI-Shenzhen, Shenzhen, China. ${ }^{8}$ Department of Chemistry, Seoul National University, Seoul, South Korea. ${ }^{9}$ Knight Cardiovascular Institute, Oregon Health \& Science University, Portland, OR, USA. ${ }^{10}$ Division of Reproductive Endocrinology and Infertility, Department of Obstetrics and Gynecology, Oregon Health \& Science University, Portland, OR, USA. ${ }^{11}$ Present address: UT Southwestern Medical Center, Molecular Biology Department, Dallas, TX, USA. ${ }^{12}$ Present address: Stem Cell Center, ASAN Institute for Life Sciences, ASAN Medical Center, Seoul, South Korea. ${ }^{13}$ Present address: Center for Reproductive Medicine, Department of Obstetrics and Gynecology, the First Affiliated Hospital of Anhui Medical University, Anhui Province Key Laboratory of Reproductive Health and Genetics, Anhui Medical University, Anhui, China.*e-mail: mitalipo@ohsu.edu

1. Adikusuma, F. et al. Large deletions induced by Cas 9 cleavage. Nature 560 , https://doi.org/10.1038/s41586-018-0380-z (2018).

2. Egli, D. et al. Inter-homologue repair in fertilized human eggs? Nature $\mathbf{5 6 0}$ https://doi.org/10.1038/s41586-018-0379-5 (2018).

3. $\mathrm{Ma}, \mathrm{H}$. et al. Correction of a pathogenic gene mutation in human embryos. Nature 548, 413-419 (2017).

4. Capmany, G., Taylor, A., Braude, P. R. \& Bolton, V. N. The timing of pronuclear formation, DNA synthesis and cleavage in the human 1-cell embryo. Mol. Hum. Reprod. 2, 299-306 (1996).

5. Lemmen, J. G., Agerholm, I. \& Ziebe, S. Kinetic markers of human embryo quality using time-lapse recordings of IVF/ICSI-fertilized oocytes. Reprod. Biomed. Online 17, 385-391 (2008).
6. Shin, H. Y. et al. CRISPR/Cas9 targeting events cause complex deletions and insertions at 17 sites in the mouse genome. Nat. Commun. 8, 15464 (2017).

7. Fogarty, N. M. E. et al. Genome editing reveals a role for OCT4 in human embryogenesis. Nature 550, 67-73 (2017).

8. Joyce, E. F., Erceg, J. \& Wu, C. T. Pairing and anti-pairing: a balancing act in the diploid genome. Curr. Opin. Genet. Dev. 37, 119-128 (2016).

9. Rong, Y. S. \& Golic, K. G. The homologous chromosome is an effective template for the repair of mitotic DNA double-strand breaks in Drosophila. Genetics 165, 1831-1842 (2003).

10. Filler Hayut, S., Melamed Bessudo, C. \& Levy, A. A. Targeted recombination between homologous chromosomes for precise breeding in tomato. Nat. Commun. 8, 15605 (2017).

11. Wu, Y. et al. Correction of a genetic disease in mouse via use of CRISPR-Cas9. Cell Stem Cell 13, 659-662 (2013).

12. Liang, P. et al. CRISPR/Cas9-mediated gene editing in human tripronuclear zygotes. Protein Cell 6, 363-372 (2015).

13. Wilde, J. J. et al. RAD51 enhances zygotic interhomolog repair. Preprint at https://doi.org/10.1101/263699 (2018).

14. Tachibana, M. et al. Generation of chimeric rhesus monkeys. Cell 148 285-295 (2012).

15. Chin, M. H. et al. Induced pluripotent stem cells and embryonic stem cells are distinguished by gene expression signatures. Cell Stem Cell 5, 111-123 (2009).

16. McKenna, A. et al. The Genome Analysis Toolkit: a MapReduce framework for analyzing next-generation DNA sequencing data. Genome Res. 20, 1297-1303 (2010)

17. Van der Auwera, G. A. et al. From FastQ data to high confidence variant calls: the Genome Analysis Toolkit best practices pipeline. Curr. Protoc. Bioinformatics 43, 11.10.1-11.10.33 (2013).

18. DePristo, M. A. et al. A framework for variation discovery and genotyping using next-generation DNA sequencing data. Nat. Genet. 43, 491-498 (2011).

Acknowledgements The authors acknowledge the OHSU Institutional Review Board (IRB), Innovative Research Advisory Panel (IRAP), Scientific Review Committee (SRC) and Data Safety Monitoring Committee (DSMC) for oversight and guidance on this study. We thank the Sequencing Core of the Vollum Institute at OHSU for assistance with Sanger sequencing. Studies conducted at OHSU were supported by the OHSU institutional funds. Work in the laboratory of J.S.K. was supported by the Institute for Basic Science (IBS-R021-D1). Work in the laboratory of J.C.I.B. was supported by the G. Harold and Leila Y. Mathers Charitable Foundation, the Moxie Foundation and the Leona M. and Harry B. Helmsley Charitable Trust. Work at BGI was supported by the Shenzhen Municipal Government of China (DRC-SZ [2016] 884).

Author contributions S.M. and H.M. conceived the study and designed the experiments. S.W.P., J.S.K. and K.S. designed CRISPR-Cas9 constructs. H.M., N.M.G., T.H., H.D., C.V.D., Y.Li., A.K., D.L., R.A., Y.L., E.K. and D.J. performed the DNA extractions, PCR and Sanger sequencing. S.W.P., A.R.P., D.K., S.T.K. and J.S.K performed WGS and analyses. J.W., K.S., Y.G., J.H.G., X.X. and J.C.I.B. performed WES and analyses. P.A., D.B., D.M.L., S.A.K., S.H., S.K. and D.H.W. coordinated recruitment of gamete donors. H.M., N.M.G. and S.M. analysed the data. H.M., D.P.W., N.M.G. and S.M. wrote the manuscript.

Competing interests Declared none.

Additional information

Extended data is available for this Reply.

Supplementary information is available for this Reply.

Reprints and permissions information is available at http://www.nature.com/ reprints.

Correspondence and requests for materials should be addressed to S.M.

https://doi.org/10.1038/s41586-018-0381-y 


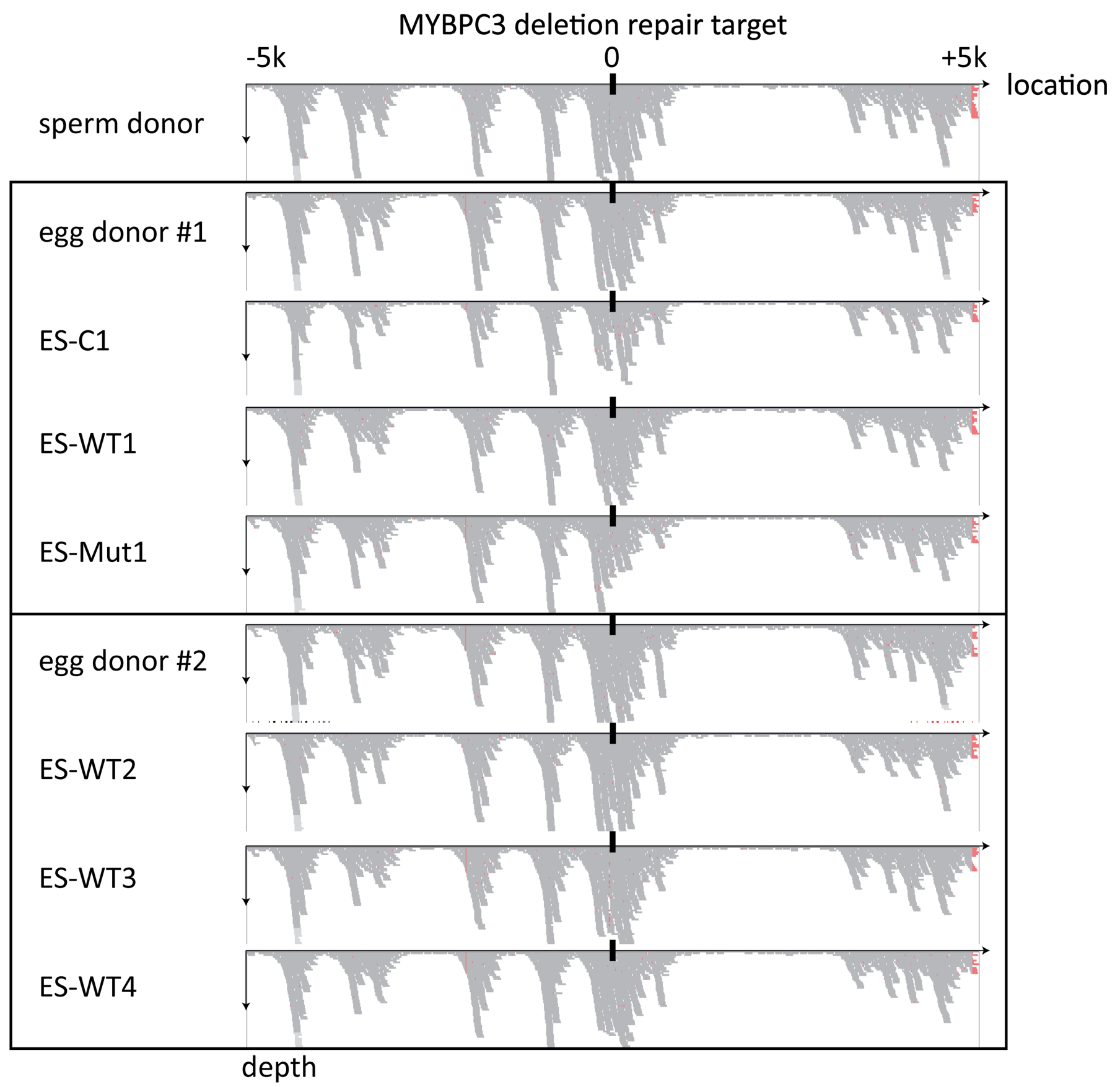

Extended Data Fig. 1 | Sequencing depth comparison of chromosome 11 in ES cell lines. Sequencing depth comparison of the chromosome 11 segment $5 \mathrm{~kb}$ downstream and $5 \mathrm{~kb}$ upstream from the MYBPC 3 mutation site in 6 ES cell lines derived from M-phase-injected embryos. 


\section{BRIEF COMMUNICATIONS ARISING}

a

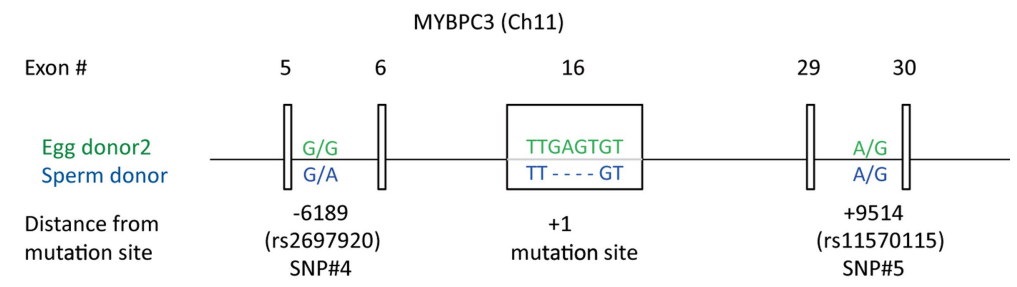

b

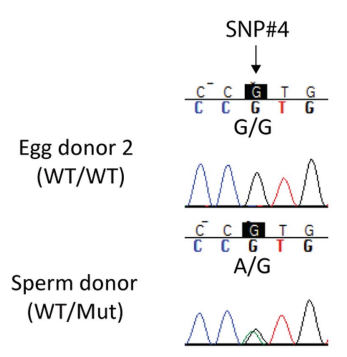

$$
\begin{array}{ccccc}
C & C & G & T & G \\
\hline C & C & G & T & G \\
& G / G &
\end{array}
$$
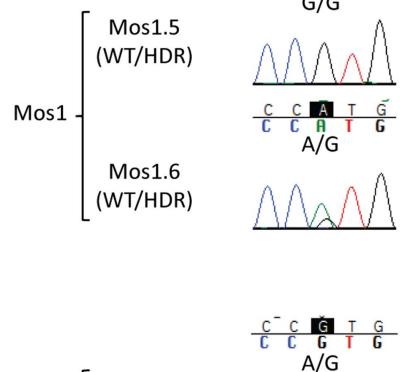

Mos7 $\left[\begin{array}{c}\text { Mos7.1 } \\ \text { (WT/HDR) } \\ \\ \text { Mos7.3 } \\ \text { (WT/HDR) }\end{array}\right.$

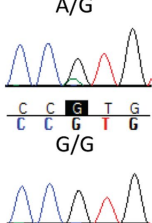

Extended Data Fig. 2 Evaluation of HDR repair and conversion tract in mosaic human embryos produced from egg donor 2 . a, Schematic map of two SNP sites within a genomic region of the MYBPC3 gene. The rs number under each SNP represents a reference number recorded at
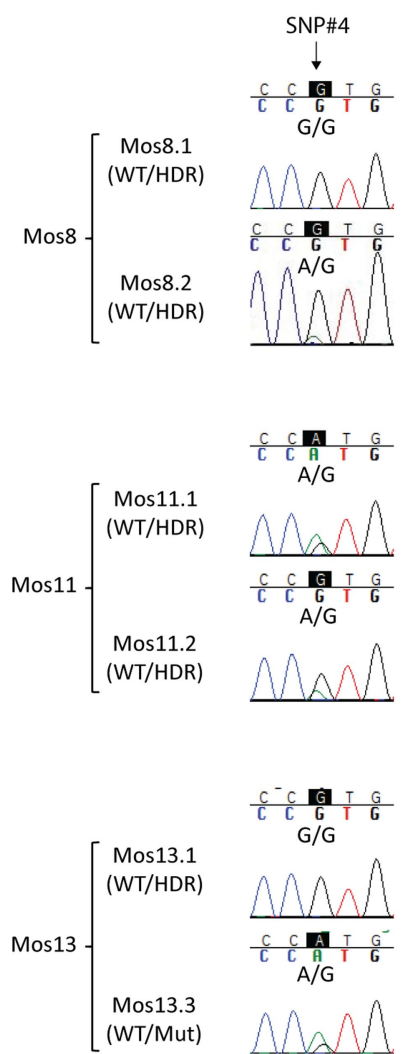

NCBI dbSNP (the Short Genetic Variation database). b, Representative chromatographs of SNP genotypes in individual blastomeres $(n=26)$ from S-phase-injected mosaic embryos. Similar results were obtained from two independent Sanger sequencing for each sample presented in $\mathbf{b}$. 


\section{BRIEF COMMUNICATIONS ARISING}

Extended Data Table 1 | MYBPC3 primer sequences used for long-range PCR

\begin{tabular}{|c|c|c|c|}
\hline PCR & PCR fragment size & Primer & Sequence $\left(5^{\prime}-3^{\prime}\right)$ \\
\hline \multirow{2}{*}{ PCR1 } & \multirow{2}{*}{ 493bp } & Forward & CCCACCCAGGTACATCTTTG \\
\hline & & Reverse & TGATCAGGTGGTGTCTCTGC \\
\hline \multirow{2}{*}{ PCR2 } & \multirow{2}{*}{$1032 b p$} & Forward & CCCACCCAGGTACATCTTTG \\
\hline & & Reverse & TCTGCGATGCTCTGGTACAC \\
\hline \multirow{2}{*}{ PCR3 } & \multirow{2}{*}{$1742 b p$} & Forward & GGCGGCACAGAGGGGATT \\
\hline & & Reverse & TGGGACACCTTTATGCGGCT \\
\hline \multirow{2}{*}{ PCR4 } & \multirow{2}{*}{$2000 b p$} & Forward & GAGACAAGGAGGTGGGTTGA \\
\hline & & Reverse & TCTGCGATGCTCTGGTACAC \\
\hline \multirow{2}{*}{ PCR5 } & \multirow{2}{*}{ 2944bp } & Forward & GAGAGGACCCCAAGGAAGAC \\
\hline & & Reverse & CGTCAATGGTCAGTTTGTGG \\
\hline \multirow{2}{*}{ PCR6 } & \multirow{2}{*}{ 4986bp } & Forward & TCCACACCCAAAAGAAAAGG \\
\hline & & Reverse & GAAACTGAGGCTCACAGAGATT \\
\hline \multirow{2}{*}{ PCR7 } & \multirow{2}{*}{ 5727bp } & Forward & GTCCGGCTTAGCACAGAGAC \\
\hline & & Reverse & GGTTCCCTGGCCAAATAAAT \\
\hline \multirow{2}{*}{ PCR8 } & \multirow{2}{*}{ 10160bp } & Forward & CCCAGAGCTGCAGGGTCTAC \\
\hline & & Reverse & ACCCTGGGTTGAAGGGTGTG \\
\hline
\end{tabular}




\section{BRIEF COMMUNICATIONS ARISING}

Extended Data Table 2 | List of WT/HDR blastomeres from S-phase-injected mosaic embryos tested for large deletions with long-range PCR

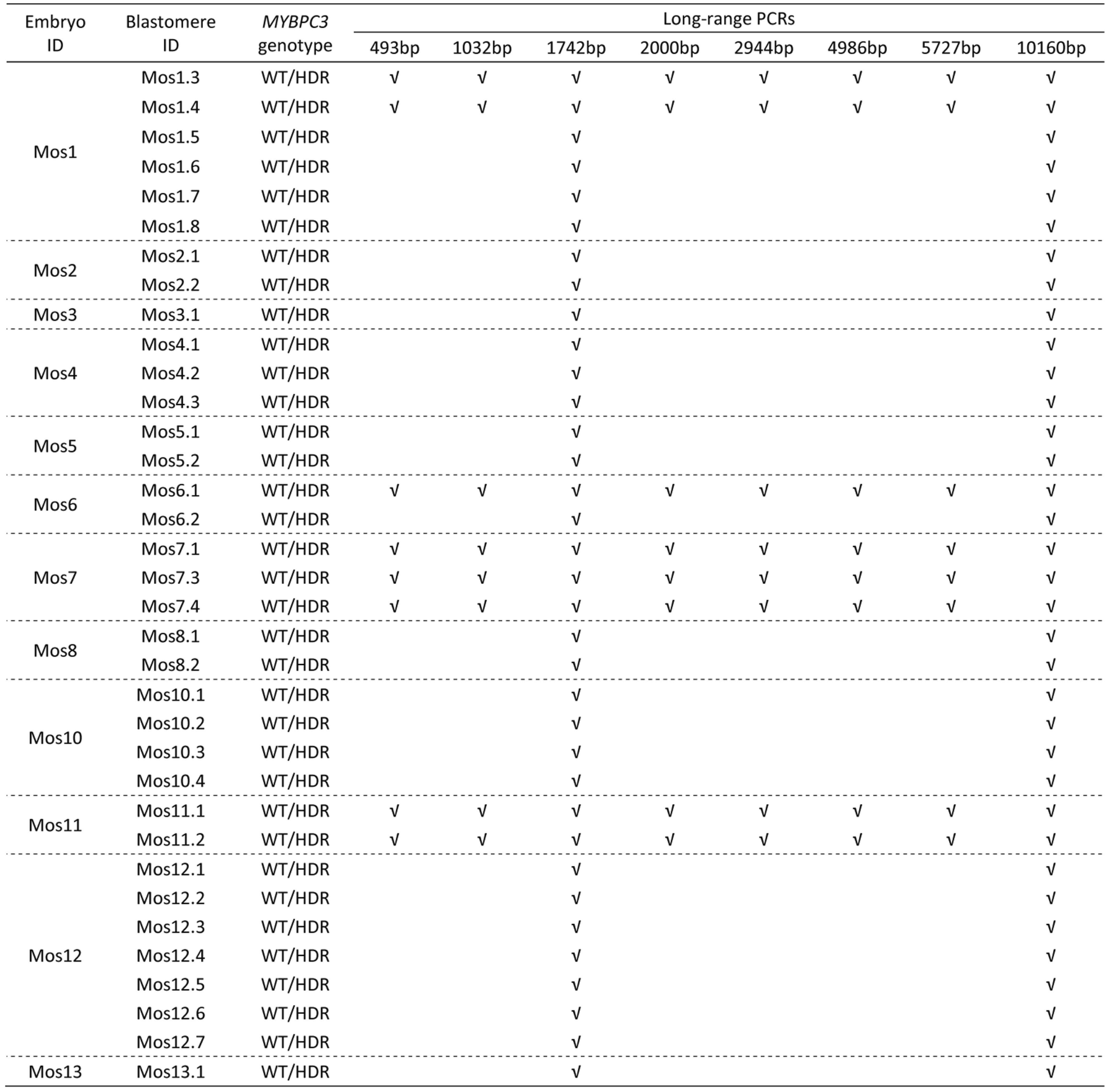




\section{BRIEF COMMUNICATIONS ARISING}

Extended Data Table 3 | List of WT/WT blastomeres from non-injected control embryos tested for large deletions with long-range PCR

\begin{tabular}{|c|c|c|c|c|c|c|c|c|c|c|}
\hline \multirow{2}{*}{ Embryo ID } & \multirow{2}{*}{$\begin{array}{c}\text { Blastomere } \\
\text { ID }\end{array}$} & \multirow{2}{*}{$\begin{array}{l}\text { MYBPC3 } \\
\text { genotype }\end{array}$} & \multicolumn{8}{|c|}{ Long-range PCRs } \\
\hline & & & $493 b p$ & $1032 b p$ & $1742 \mathrm{bp}$ & $2000 \mathrm{bp}$ & $2944 b p$ & $4986 b p$ & $5727 \mathrm{bp}$ & $10160 \mathrm{bp}$ \\
\hline $\mathrm{C} 1$ & C1.1 & WT/WT & & & $\sqrt{ }$ & & & & & $\mathrm{V}$ \\
\hline \multirow{2}{*}{$\mathrm{C} 2$} & $\mathrm{C} 2.2$ & WT/WT & V & $\sqrt{ }$ & & V & V & $\sqrt{ }$ & V & \\
\hline & $\mathrm{C} 2.3$ & WT/WT & $\sqrt{ }$ & $\sqrt{ }$ & & $\mathrm{V}$ & 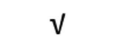 & $\sqrt{ }$ & V & \\
\hline \multirow{4}{*}{$\mathrm{C} 17$} & C17.1 & WT/WT & $v$ & $\checkmark$ & & v & v & v & $v$ & \\
\hline & $\mathrm{C} 17.2$ & WT/WT & v & $\sqrt{ }$ & & v & v & v & v & \\
\hline & C17.3 & WT/WT & v & $\sqrt{ }$ & & v & v & v & v & \\
\hline & C17.4 & WT/WT & $\sqrt{ }$ & $\sqrt{ }$ & & v & $v$ & $\sqrt{ }$ & V & \\
\hline
\end{tabular}


Extended Data Table 4 | List of blastomeres from M-phase-injected embryos tested for large deletions with long-range PCR

\begin{tabular}{|c|c|c|c|c|}
\hline Embryo ID & Blastomere ID & MYBPC3 genotype & PCR 1742bp & PCR 10160bp \\
\hline M2-WT1 & M2-WT1.2 & WT/WT & v & v \\
\hline M2-WT2 & M2-WT2.3 & WT/WT & v & v \\
\hline M2-WT3 & M2-WT3.2 & WT/WT & V & $\checkmark$ \\
\hline M2-WT4 & M2-WT4.2 & WT/WT & $\sqrt{ }$ & 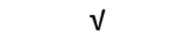 \\
\hline M2-WT5 & M2-WT5.2 & WT/WT & $\checkmark$ & $v$ \\
\hline M2-WT6 & M2-WT6.2 & WT/WT & v & v \\
\hline M2-WT7 & M2-WT7.2 & WT/WT & $\checkmark$ & $v$ \\
\hline M2-WT8 & M2-WT8.2 & WT/WT & $\checkmark$ & $\checkmark$ \\
\hline M2-WT9 & M2-WT9.3 & WT/WT & $v$ & $v$ \\
\hline M2-WT10 & M2-WT10.2 & WT/WT & V & V \\
\hline M2-WT11 & M2-WT11.2 & WT/WT & $\checkmark$ & $v$ \\
\hline M2-WT12 & M2-WT12.5 & WT/WT & V & v \\
\hline M2-WT13 & M2-WT13.2 & WT/WT & 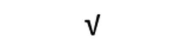 & 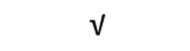 \\
\hline M2-WT14 & M2-WT14.2 & WT/WT & v & v \\
\hline M2-WT15 & M2-WT15.2 & WT/WT & $\checkmark$ & $v$ \\
\hline M2-WT16 & M2-WT16.2 & WT/WT & $\checkmark$ & $v$ \\
\hline M2-WT17 & M2-WT17.2 & WT/WT & v & v \\
\hline M2-WT18 & M2-WT18.2 & WT/WT & $\checkmark$ & $\checkmark$ \\
\hline M2-WT19 & M2-WT19.2 & WT/WT & $v$ & $v$ \\
\hline M2-WT20 & M2-WT20.2 & WT/WT & $\checkmark$ & $\checkmark$ \\
\hline M2-WT21 & M2-WT21.4 & WT/WT & V & v \\
\hline M2-WT22 & M2-WT22.3 & WT/WT & 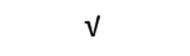 & V \\
\hline M2-WT23 & M2-WT23.4 & WT/WT & v & v \\
\hline M2-WT24 & M2-WT24.4 & WT/WT & $v$ & $v$ \\
\hline M2-WT25 & M2-WT25.2 & WT/WT & V & V \\
\hline M2-WT26 & M2-WT26.3 & WT/WT & $v$ & $v$ \\
\hline M2-WT27 & M2-WT27.2 & WT/WT & $v$ & $v$ \\
\hline M2-WT28 & M2-WT28.2 & WT/WT & $v$ & v \\
\hline M2-WT29 & M2-WT29.2 & WT/WT & $v$ & $v$ \\
\hline M2-WT30 & M2-WT30.2 & WT/WT & V & V \\
\hline M2-WT31 & M2-WT31.3 & WT/WT & $\checkmark$ & $\checkmark$ \\
\hline M2-WT32 & M2-WT32.2 & WT/WT & $v$ & $v$ \\
\hline M2-WT33 & M2-WT33.2 & WT/WT & 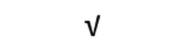 & V \\
\hline M2-WT34 & M2-WT34.4 & WT/WT & $v$ & $v$ \\
\hline M2-WT35 & M2-WT35.2 & WT/WT & $\checkmark$ & $\checkmark$ \\
\hline M2-WT36 & M2-WT36.3 & WT/WT & v & v \\
\hline M2-WT37 & M2-WT37.2 & WT/WT & $\checkmark$ & $\checkmark$ \\
\hline M2-WT38 & M2-WT38.2 & WT/WT & $v$ & $v$ \\
\hline M2-WT39 & M2-WT39.3 & WT/WT & $\checkmark$ & V \\
\hline
\end{tabular}


Extended Data Table 5 | Parentage analysis of ES cell lines derived from M-phase-injected embryos determined by STR assay

\begin{tabular}{|c|c|c|c|c|c|c|c|c|c|c|c|}
\hline STR loci & ES-C1 & ES-WT1 & ES-Mut1 & $\begin{array}{r}\text { Oocyte } \\
\text { donor1 }\end{array}$ & $\begin{array}{c}\text { Sperm } \\
\text { donor }\end{array}$ & ES-WT2 & ES-WT3 & $\begin{array}{c}\text { ES- } \\
\text { WT4 }\end{array}$ & $\begin{array}{c}\text { ES- } \\
\text { Mut2 }\end{array}$ & $\begin{array}{l}\text { Oocyte } \\
\text { donor2 }\end{array}$ & $\begin{array}{c}\text { Sperm } \\
\text { donor }\end{array}$ \\
\hline $\begin{array}{c}\text { Sex } \\
\text { AME } \\
\end{array}$ & $\begin{array}{c}\mathbf{M} \\
\mathbf{X Y}\end{array}$ & $\begin{array}{c}\mathbf{F} \\
\mathbf{X X} \\
\end{array}$ & $\begin{array}{c}\mathbf{F} \\
\mathbf{X X} \\
\end{array}$ & $\begin{array}{c}\mathbf{F} \\
\mathbf{X X} \\
\end{array}$ & $\begin{array}{c}\mathbf{M} \\
\mathbf{X Y}\end{array}$ & $\begin{array}{c}\mathbf{F} \\
\mathbf{X X}\end{array}$ & $\begin{array}{c}\text { M } \\
\mathbf{X Y} \\
\end{array}$ & $\begin{array}{c}\mathbf{F} \\
\mathbf{X X} \\
\end{array}$ & $\begin{array}{c}\mathbf{F} \\
\mathbf{X X} \\
\end{array}$ & $\begin{array}{c}\mathbf{F} \\
\mathbf{X X} \\
\end{array}$ & $\begin{array}{c}\mathbf{M} \\
\mathbf{X Y}\end{array}$ \\
\hline D1S548 & $168 / 172$ & $168 / 172$ & $152 / 172$ & $152 / 168$ & $172 / 172$ & $152 / 172$ & $152 / 172$ & $172 / 176$ & $172 / 176$ & $152 / 176$ & $172 / 172$ \\
\hline D2S1333 & $285 / 293$ & $285 / 285$ & $285 / 301$ & $285 / 301$ & $285 / 293$ & $285 / 293$ & $293 / 297$ & $285 / 293$ & $285 / 297$ & $285 / 297$ & $285 / 293$ \\
\hline D3S1768 & $184 / 188$ & $192 / 196$ & $192 / 196$ & $188 / 196$ & $184 / 192$ & $184 / 204$ & $184 / 196$ & $184 / 204$ & $192 / 204$ & $196 / 204$ & $184 / 192$ \\
\hline D4S2365 & $296 / 300$ & $296 / 300$ & $296 / 296$ & $296 / 300$ & $296 / 296$ & * & $296 / 296$ & $296 / 296$ & $296 / 296$ & $296 / 296$ & $296 / 296$ \\
\hline D4S413 & $123 / 125$ & $123 / 125$ & $123 / 125$ & $123 / 123$ & $123 / 125$ & $125 / 125$ & $125 / 125$ & $123 / 125$ & $123 / 125$ & $125 / 125$ & $123 / 125$ \\
\hline D5S1457 & $101 / 123$ & $123 / 123$ & $123 / 123$ & $123 / 123$ & $101 / 123$ & $123 / 123$ & $123 / 123$ & $101 / 123$ & $101 / 101$ & $101 / 123$ & $101 / 123$ \\
\hline D6S276 & $235 / 251$ & $227 / 251$ & $227 / 251$ & $227 / 235$ & $251 / 251$ & $227 / 251$ & $251 / 251$ & $251 / 251$ & $251 / 251$ & $227 / 251$ & $251 / 251$ \\
\hline D6S501 & $172 / 172$ & $168 / 172$ & $168 / 173$ & $168 / 172$ & $172 / 173$ & $172 / 172$ & $173 / 176$ & $172 / 172$ & $172 / 172$ & $172 / 176$ & $172 / 173$ \\
\hline D11S2002 & $254 / 254$ & $254 / 254$ & $254 / 254$ & $254 / 254$ & $254 / 254$ & $254 / 254$ & $254 / 254$ & $254 / 254$ & $254 / 254$ & $254 / 254$ & $254 / 254$ \\
\hline D11S925 & $299 / 305$ & 299/305 & $299 / 305$ & $305 / 305$ & $282 / 299$ & $299 / 305$ & $282 / 305$ & $299 / 301$ & $282 / 305$ & $301 / 305$ & $282 / 299$ \\
\hline D12S364 & $266 / 272$ & $266 / 270$ & $266 / 270$ & $270 / 272$ & $266 / 274$ & $274 / 280$ & $274 / 280$ & $266 / 280$ & $266 / 280$ & $272 / 280$ & $266 / 274$ \\
\hline D12S67 & $252 / 252$ & $252 / 260$ & $252 / 260$ & $252 / 260$ & $252 / 264$ & $252 / 264$ & $252 / 252$ & $252 / 256$ & $256 / 264$ & $252 / 256$ & $252 / 264$ \\
\hline D13S765 & $188 / 196$ & $188 / 196$ & $188 / 196$ & $188 / 200$ & $192 / 196$ & $192 / 200$ & $192 / 200$ & $196 / 200$ & $196 / 200$ & $188 / 200$ & $192 / 196$ \\
\hline D16S403 & $139 / 145$ & $139 / 145$ & $145 / 145$ & $145 / 145$ & $139 / 145$ & $139 / 145$ & * & $145 / 145$ & $139 / 145$ & $143 / 145$ & $139 / 145$ \\
\hline D17S1300 & $257 / 269$ & $257 / 269$ & $257 / 269$ & $257 / 269$ & $257 / 257$ & $257 / 269$ & $257 / 269$ & $257 / 269$ & $257 / 265$ & $265 / 269$ & $257 / 257$ \\
\hline D18S537 & $196 / 208$ & 196/196 & $196 / 196$ & $196 / 208$ & $196 / 196$ & $196 / 196$ & 196/196 & $196 / 196$ & 196/196 & 196/196 & $196 / 196$ \\
\hline D18S72 & $301 / 305$ & $301 / 305$ & $305 / 305$ & $301 / 305$ & $305 / 305$ & $303 / 305$ & $305 / 305$ & $305 / 305$ & $303 / 305$ & $303 / 305$ & $305 / 305$ \\
\hline D22S685 & $192 / 200$ & $192 / 200$ & $180 / 180$ & $180 / 192$ & $180 / 200$ & $180 / 192$ & $180 / 196$ & $192 / 200$ & $180 / 192$ & $192 / 196$ & $180 / 200$ \\
\hline DXS2506 & 286 & $278 / 286$ & $278 / 282$ & $282 / 286$ & 278 & $278 / 282$ & 282 & $278 / 282$ & $278 / 282$ & $282 / 282$ & 278 \\
\hline MFGT22 & $104 / 108$ & $108 / 108$ & $104 / 112$ & $108 / 112$ & $104 / 108$ & $106 / 108$ & $106 / 108$ & $104 / 106$ & $106 / 108$ & $104 / 106$ & $104 / 108$ \\
\hline
\end{tabular}




\section{natureresearch}

Corresponding author(s): Shoukhrat Mitalipov

$\square$ Initial submission $\square$ Revised version $\quad \mathrm{X}$ Final submission

\section{Life Sciences Reporting Summary}

Nature Research wishes to improve the reproducibility of the work that we publish. This form is intended for publication with all accepted life science papers and provides structure for consistency and transparency in reporting. Every life science submission will use this form; some list items might not apply to an individual manuscript, but all fields must be completed for clarity.

For further information on the points included in this form, see Reporting Life Sciences Research. For further information on Nature Research policies, including our data availability policy, see Authors \& Referees and the Editorial Policy Checklist.

\section{- Experimental design}

\section{Sample size}

Describe how sample size was determined.

2. Data exclusions

Describe any data exclusions.

3. Replication

Describe whether the experimental findings were reliably reproduced.

\section{Randomization}

Describe how samples/organisms/participants were allocated into experimental groups.

5. Blinding whether the investigators were blinded to group allocation during data collection and/or analysis.
All samples presented in this study were from our previous Nature publication and sample size was determined based on specific BCA response.

No data were excluded

Human embryo HDR and mosaicism findings were reproduced with multiple oocyte donations from different donors. On-target genotyping by Sanger were validated by STR independently.

Sperm containing mutant and WT MYBPC3 was randomly injected into oocytes collected from different donors. CRISPR-Cas 9 was randomly injected into zygotes.

During all on-target and off-target PCR and Sanger sequencing, the personnel was blinded regarding the sample origin.

Note: all studies involving animals and/or human research participants must disclose whether blinding and randomization were used.

6. Statistical parameters

For all figures and tables that use statistical methods, confirm that the following items are present in relevant figure legends (or in the Methods section if additional space is needed).

n/a $\mid$ Confirmed

Х $\square$ The exact sample size $(n)$ for each experimental group/condition, given as a discrete number and unit of measurement (animals, litters, cultures, etc.)

$\mathrm{X}$ A description of how samples were collected, noting whether measurements were taken from distinct samples or whether the same

$\mathrm{X}$ sample was measured repeatedly

X A statement indicating how many times each experiment was replicated

$\triangle$ The statistical test(s) used and whether they are one- or two-sided (note: only common tests should be described solely by name; more

$\square$ complex techniques should be described in the Methods section)

$\bigotimes \square$ A description of any assumptions or corrections, such as an adjustment for multiple comparisons

Х $\square$ The test results (e.g. $P$ values) given as exact values whenever possible and with confidence intervals noted

Х $\square$ A clear description of statistics including central tendency (e.g. median, mean) and variation (e.g. standard deviation, interquartile range)

Х $\square$ Clearly defined error bars 
Policy information about availability of computer code

\section{Software}

Describe the software used to analyze the data in this study.

Sequencher v5.0 (GeneCodes) was used for Sanger sequencing. SOPAnuke (1.5.2), Burrows-Wheeler Aligner (BWA) (0.7.12), Picard v1.54, GATK (3.3.0) and

AnnoDB v3 were used for WES and WGS data analysis.

For manuscripts utilizing custom algorithms or software that are central to the paper but not yet described in the published literature, software must be made available to editors and reviewers upon request. We strongly encourage code deposition in a community repository (e.g. GitHub). Nature Methods guidance for providing algorithms and software for publication provides further information on this topic.

\section{- Materials and reagents}

Policy information about availability of materials

8. Materials availability

Indicate whether there are restrictions on availability of unique materials or if these materials are only available for distribution by a for-profit company.

\section{Antibodies}

Describe the antibodies used and how they were validated for use in the system under study (i.e. assay and species).

10. Eukaryotic cell lines

a. State the source of each eukaryotic cell line used.

b. Describe the method of cell line authentication used.

c. Report whether the cell lines were tested for mycoplasma contamination.

d. If any of the cell lines used are listed in the database of commonly misidentified cell lines maintained by ICLAC, provide a scientific rationale for their use.
ESCs from this study are available for distribution following MTA, dependent upon IRB and DSMC approval.

No antibodies were used in this study.

All ESC and iPSC lines included in the study were generated in this study.

WGS, WES, Sanger sequencing, STR.

All cell lines were negative for mycoplasma contamination.

No commonly misidentified cell lines were used in this study.

\section{- Animals and human research participants}

Policy information about studies involving animals; when reporting animal research, follow the ARRIVE guidelines

11. Description of research animals

Provide details on animals and/or animal-derived materials used in the study.

No animals were used in this study.

Policy information about studies involving human research participants

\section{Description of human research participants}

Describe the covariate-relevant population characteristics of the human research participants.
A sperm donor carrying MYBPC 3 gene mutation and healthy oocyte donors with age between 21 to 35 years old were recruited for this study. 